\title{
Resolvent Systems of Difference Polynomial Ideals
}

\author{
Xiao-Shan Gao and Chun-Ming Yuan \\ Key Laboratory of Mathematics Mechanization \\ Institute of Systems Science, AMSS, Academia Sinica \\ Beijing 100080, China \\ (xgao,cmyuan)@mmrc.iss.ac.cn
}

\begin{abstract}
In this paper, a new theory of resolvent systems is developed for prime difference ideals and difference ideals defined by coherent and proper irreducible ascending chains. Algorithms to compute such resolvent systems are also given. As a consequence, we prove that any irreducible difference variety is birationally equivalent to an irreducible difference variety of codimension one. As a preparation to the resolvent theory, we also prove that the saturation ideal of a coherent and proper ascending chain is unmixed in the sense that all its prime components have the same dimension and order.
\end{abstract}

Keywords. Resolvent, difference ascending chain, difference polynomial, difference variety, unmixed decomposition.

\section{Introduction}

A classic result in algebraic geometry states that any irreducible variety is birationally equivalent to an irreducible hypersurface. Or equivalently, any finitely generated algebraic extension field can be generated with a single element, called the primitive element of the extension field. Algorithms to construct such hypersurfaces or primitive elements were proposed based on the methods of resultant computation by Trager [16] and Loos [10], the Gröbner basis method by Gianni and Mora [9], Kobayashi et al [9], and Yokoyama et al [20], and the characteristic set method by Gao-Chou [5, 7] and Wang-Lin [17]. The idea is to introduce a linear transformation of variables and show that the new equation system can be transformed into the following special form using various elimination theories

$$
R\left(x_{0}\right), I_{1}\left(x_{0}\right) x_{1}+U_{1}\left(x_{0}\right), \ldots, I_{n}\left(x_{0}\right) x_{n}+U_{n}\left(x_{0}\right)
$$

where $R, I_{i}, U_{i}$ are univariate polynomials in $x_{0}$.

In [14], Ritt proved similar results for ordinary differential polynomial equation systems by introducing the concept of resolvents for a prime ideal. Kolchin further gave generalizations of the resolvent to the partial differential case [12]. In [1], Cluzeau and Hubert extended the concept of resolvent to regular differential ideals and proposed an algorithm to compute it.

Following the work of Ritt[15], Cohn established the Difference Algebra [2]. Recently, elimination theories for difference polynomial systems were studied by Mansfield and Szanto [13], van der Hoeven [18], and Gao-Luo [8]. The concept of resolvent for an irreducible 
difference variety was also introduced by Cohn $[2,3]$. In difference case, the resolvent is not a single difference polynomial anymore. In general, it is an irreducible difference variety of codimension one, which may be called the resolvent variety. But, the difference resolvent theory is not as complete as the algebraic and differential cases. First, when establishing the birational equivalence between an irreducible variety $V$ and its resolvent variety $W$, the operations of inversion need to be used. More precisely, the rational map is from $W$ to $\mathbf{E}^{-t} V$ where $\mathbf{E}$ is difference operator and $t$ an integer. Second, no algorithms were given to compute the resolvents.

In this paper, a more complete difference resolvent theory is proposed. We prove that for an irreducible difference variety $V$, there always exists a resolvent variety $W$ such that there is a birational equivalence between $V$ and $W$. This is possible, because we prove that an irreducible difference variety can be represented by a coherent and strong irreducible ascending chain [8]. From this chain, a resolvent property with better properties is developed. We also give an algorithm to compute a coherent and strong irreducible ascending chain which represents the resolvent variety. Furthermore, for a coherent and proper irreducible ascending chain (definition in Section 3), we gave an algorithm to construct a system of resolvent varieties. In [8], we give an algorithm to decompose the zero set of a set of difference polynomials into the zero sets of difference varieties represented by coherent and proper irreducible ascending chains. Combining this result and the result in this paper, it is always possible to represent the zero set of a difference polynomial system by a series of resolvent varieties. A consequence of the resolvent system is that we reduce the test of the strong irreducibility for an ascending chain to the test of the strong irreducibility for an ascending chain in one variable.

In order to establish the resolvent theory, we prove a basic property of a coherent and proper irreducible ascending chain, which says that the saturation ideal of a coherent and proper irreducible ascending chain is unmixed in the sense that all its prime components have the same dimension and order.

Based on the implementation of a characteristic set method for difference polynomial systems introduced in [8], we implement the algorithms proposed in this paper. Examples are given to illustrate the algorithms. The most time consuming part of the computation process is the computation of the characteristic set.

The rest of this paper is organized as follows. In Section 2, we introduce some notations and preliminary results. In Section 3, we prove the unmixed property of a coherent and proper irreducible ascending chain. In Section 4, the theory and algorithm for the resolvent variety of prime ideals are developed. In Section 5, the theory and algorithm for coherent and proper irreducible chains are introduced. In Section 6, conclusions are presented.

\section{Difference fields, difference polynomials, and difference ideals}

A difference field $\mathcal{F}$ is a field with a third unitary operation $\mathbf{E}$ satisfying: for any $a, b \in \mathcal{F}$, $\mathbf{E}(a+b)=\mathbf{E} a+\mathbf{E} b, \mathbf{E}(a b)=\mathbf{E} a \cdot \mathbf{E} b$, and $\mathbf{E} a=0$ if and only if $a=0$. Here, $\mathbf{E}$ is called the transforming operator or simply a transform of $\mathcal{F}$. If $a \in \mathcal{F}, \mathbf{E} a$ is called the transform of $a . \mathbf{E}^{n} a=\mathbf{E}\left(\mathbf{E}^{n-1} a\right)$ is known as the n'th transform. If $\mathbf{E}^{-1} a$ is defined for all $a \in \mathcal{F}$, we say that $\mathcal{F}$ is inversive. Every difference field has an inversive closure [2]. In this paper, all difference fields are assumed to be inversive and of characteristic zero. 
As an example, let $\mathcal{K}$ be the set of rational functions in variable $x$ defined on the complex plane. Let $\mathbf{E}$ be the map: $\mathbf{E} f(x)=f(x+1), f \in \mathcal{K}$. Then $\mathcal{K}$ is a difference field with transforming operator $\mathbf{E}$. This is an inversive field. In this paper, $\mathcal{K}$ is assumed to be this difference field.

Let $\mathbb{X}=\left\{x_{1}, \ldots, x_{n}\right\}$ be indeterminants. Then $\mathcal{K}\{\mathbb{X}\}=\mathcal{K}\left\{x_{1}, \ldots, x_{n}\right\}$ is called an $n$ fold difference polynomial (abbr. r-pol) ring over $\mathcal{K}$. Any r-pol $P$ in $\mathcal{K}\{\mathbb{X}\}$ is an ordinary polynomial in variables $\mathbf{E}^{k} x_{j}(k=0,1,2, \ldots, j=1, \ldots, n)$. For convenience, we also denote $\mathbf{E}^{k} x_{j}$ by $x_{j, k}$ or $x_{j}(x+k)$.

Let $P \in \mathcal{K}\{\mathbb{X}\}$. The class of $P$, denoted by $\operatorname{class}(P)$, is the least $p$ such that $P \in$ $\mathcal{K}\left\{x_{1}, \ldots, x_{p}\right\}$. If $P \in \mathcal{K}$, we set class $(P)=0$. The $\operatorname{order}$ of $P$ w.r.t $x_{i}$, denoted by $\operatorname{ord}\left(P, x_{i}\right)$, is the largest $j$ such that $x_{i, j}$ occurs in $P$. When $x_{i, j}$ does not occur in $\mathrm{P}$, we set $\operatorname{ord}\left(P, x_{i}\right)=$ -1 . If class $(P)=p$ and $\operatorname{ord}\left(P, x_{p}\right)=q$, we called $x_{p}$ the leading variable and $x_{p, q}$ the lead of $\mathrm{P}$, denoted as $\operatorname{lvar}(P)$ and lead $(P)$, respectively. The leading coefficient of $P$ as a univariate polynomial in lead $(P)$ is called the initial of $P$, and is denoted as $\operatorname{init}(P)$.

An $n$-tuple over $\mathcal{K}$ is of the form $\mathbf{a}=\left(a_{1}, \ldots, a_{n}\right)$, where the $a_{i}$ are selected from some difference extension field of $\mathcal{K}$. Let $P \in \mathcal{K}\{\mathbb{X}\}$. To substitute an n-tuple a into $P$ means to replace each of the $x_{i, j}$ occurring in $P$ with $\mathbf{E}^{j} a_{i}$. Let $\mathbb{P}$ be a set of r-pols in $\mathcal{K}\{\mathbb{X}\}$. An n-tuple over $\mathcal{K}$ is called a solution of the equation set $\mathbb{P}=0$ if the result of substituting the n-tuple into each r-pol in $\mathbb{P}$ is zero. We use Zero $(\mathbb{P})$ to denote the set of solutions of $\mathbb{P}=0$. For an r-pol $P$, we use $\operatorname{Zero}(\mathbb{P} / P)$ to denote the set of solutions of $\mathbb{P}=0$ which are not solutions of $P=0$.

For instance, let $P=\mathbf{E} x_{1} \cdot x_{1}+\mathbf{E} x_{1}-x_{1}$. Then $x_{1}=\frac{1}{x+c(x)}$ is a solution of $P=0$, where $c(x)$ is any function satisfying $c(x+1)=c(x)$.

A field $\mathcal{K}$ is called aperiodic if there does not exist an integer $n$ such that for all $a \in \mathcal{K}$, $\mathbf{E}^{n} a=a$. It is known that (p201 [2])

Lemma 2.1 Let $\mathcal{K}$ be an aperiodic field and $P \in \mathcal{K}\{\mathbb{X}\}$ a nonzero r-pol. Then we can find an $n$-tuple $\left(\alpha_{1}, \ldots, \alpha_{n}\right) \in \mathcal{K}^{n}$ such that $P\left(\alpha_{1}, \ldots, \alpha_{n}\right) \neq 0$.

A difference ideal is a subset $I$ of $\mathcal{K}\{\mathbb{X}\}$, which is an algebraic ideal in $\mathcal{K}\{\mathbb{X}\}$ and is closed under the transform. A difference ideal $I$ is called reflexive if for an r-pol $P, \mathbf{E} P \in I$ implies $P \in I$. Let $\mathbb{P}$ be a set of elements of $\mathcal{K}\{\mathbb{X}\}$. The difference ideal generated by $\mathbb{P}$ is denoted by $[\mathbb{P}]$. The (ordinary or algebraic) ideal generated by $\mathbb{P}$ is denoted as $(\mathbb{P})$. A difference ideal $I$ is called perfect if the presence in $I$ of a product of powers of transforms of an r-pol $P$ implies $P \in I$. The perfect difference ideal generated by $\mathbb{P}$ is denoted as $\{\mathbb{P}\}$. A perfect ideal is always reflexive. A difference ideal $I$ is called a prime ideal if for r-pols $P$ and $Q, P Q \in I$ implies $P \in I$ or $Q \in I$.

Let $I \subset \mathcal{K}\{\mathbb{X}\}$ be a reflexive prime ideal. Then $I$ has a generic zero $\alpha$ which has the following property: an r-pol $P \in I$ if and only if $P(\alpha)=0$ [2]. For a reflexive prime difference ideal $I \subset \mathcal{K}\{\mathbb{X}\}$, we define the dimension of $I$ as the difference transcendental degree of a generic zero $\alpha=\left(\alpha_{1}, \ldots, \alpha_{n}\right)$ of $I$ over $\mathcal{K}[2]$. Geometrically, this is the maximal number of $\alpha_{i}$ which are not the solution of an r-pol in $I$.

Let $I$ be a difference ideal. Rename $\mathbb{X}=\left\{x_{1}, \ldots, x_{n}\right\}$ as two subsets: $\mathbb{U}=\left\{u_{1}, \ldots, u_{q}\right\}$ and $\mathbb{Y}=\left\{y_{1}, \ldots, y_{p}\right\}(p+q=n)$. $\mathbb{U}$ is called a parametric set of $I$ if $I \cap \mathcal{K}\{\mathbb{U}\}=\{0\}$ and $\forall y_{i} \in \mathbb{Y}, I \cap \mathcal{K}\left\{\mathbb{U}, y_{i}\right\} \neq\{0\}$. It is known that the dimension of a reflexive prime ideal equals to the number of its parameters. 
Let $\mathbb{U}$ be a parametric set of a reflexive prime ideal $I$. The order of $I$ w.r.t $\mathbb{U}$, denoted as $\operatorname{ord}_{\mathbb{U}} I$, is the maximum number of $y_{i j}$ which are algebraic independent over $\mathcal{K}\{\mathbb{U}\}$. More precisely, if we denote $Y_{s}$ as a set consisting of some $y_{i, j}$, then $\operatorname{ord}_{\mathbb{U}} I=\max _{I \cap \mathcal{K}\{\mathbb{U}\}\left[Y_{s}\right]=\{0\}}\left|Y_{s}\right|$. Let $\left(\beta_{1}, \ldots, \beta_{q}, \gamma_{1}, \ldots, \gamma_{p}\right)$ be a generic zero of $I$ corresponding to $\mathbb{U}$ and $\mathbb{Y}$. Then, the order of $I$ w.r.t $\mathbb{U}$ is the algebraic transcendental degree of $\left(\gamma_{1}, \ldots, \gamma_{p}\right)$ over $\mathcal{K}\left\langle\beta_{1}, \ldots, \beta_{q}\right\rangle[2]$.

The effective order of $I$ w.r.t $\mathbb{U}$ is the maximum number of $y_{i j}$ which is algebraic independent over $\mathcal{K}\{\mathbb{U}\}^{*}$, where $\mathcal{K}\{\mathbb{U}\}^{*}$ is the inversive closure of $\mathcal{K}\{\mathbb{U}\}$, and we denote it as $\operatorname{Eord}_{\mathbb{U}} I$. It is clear that $\operatorname{Eord}_{\mathbb{U}} I \leq \operatorname{ord}_{\mathbb{U}} I$.

Lemma 2.2 Let $I$ be a prime ideal with $\mathbb{U}$ as a set of parameters. If $\operatorname{Eord}_{\mathbb{U}} I=\operatorname{ord}_{\mathbb{U}} I$ then $I$ is reflexive.

Proof. We need only to prove that if $P \notin I$, then $\mathbf{E} P \notin I$. Let $o=\operatorname{ord}_{\mathbb{U}}(I)$ and $Y_{0}$ a set of $o$ elements of the form $y_{i, j}$ such that $I \cap \mathcal{K}\{\mathbb{U}\}\left[Y_{0}\right]=\{0\}$. Since $P \notin I$, there is a nonzero $M \in \mathcal{K}\{\mathbb{U}\}\left[Y_{0}\right]$ such that $P=M \bmod I$. Since $\operatorname{Eord}_{\mathbb{U}} I=\operatorname{ord}_{\mathbb{U}} I$, we have $I \cap \mathcal{K}\{\mathbb{U}\}\left[\mathbf{E} Y_{0}\right]=\{0\}$. As a consequence, $\mathbf{E} M \notin I$. Hence $\mathbf{E} P \notin I$. I

Let $\mathbb{U}$ be a parametric set of a reflexive prime ideal $I$, and $\left(\beta_{1}, \ldots, \beta_{q}, \gamma_{1}, \ldots, \gamma_{p}\right)$ a generic zero of $I$ where $\beta_{1}, \ldots, \beta_{q}$ are corresponding to $\mathbb{U}$. Then the limit degree of $I$ w.r.t $\mathbb{U}$ is defined as $\operatorname{ld}_{\mathbb{U}}(I)=\liminf _{k}\left[\mathcal{K}_{0}\left(\phi_{k}\right): \mathcal{K}_{0}\left(\phi_{k-1}\right)\right]$, where $\mathcal{K}_{0}=\mathcal{K}\left\langle\beta_{1}, \ldots, \beta_{q}\right\rangle$ and $\phi_{s}=\left\{\gamma_{i j}, 1 \leq i \leq\right.$ $p, 0 \leq j \leq s\}$.

Let $\mathcal{D}$ be a difference field with a difference operator $\mathbf{E}$. A difference kernel $R$ over $\mathcal{D}$ is an overfield $\mathcal{D}\left(a, a_{1}, \ldots, a_{r}\right), r \geq 1$, of $\mathcal{D}$, each $a_{i}$ denoting a vector $a_{i}^{(1)}, \ldots, a_{i}^{(n)}$, and an extension $\tau$ of $\mathbf{E}$ to an isomorphism of $\mathcal{D}\left(a, a_{1}, \ldots, a_{r-1}\right)$ onto $\mathcal{D}\left(a_{1}, \ldots, a_{r}\right)$, such that $\tau a_{i}=a_{i+1}, i=0,1, \ldots, r-1$. (Of course, $a_{0}=a$.) $r$ is called the length of the kernel. Let $\phi \subset a_{r}$ be a basis for transcendence independence of $a_{r}$ over $\mathcal{D}\left(a, \ldots, a_{r-1}\right)$. Then the limit degree of $R$ w.r.t $\phi$ is defined as $\mathcal{D}\left(a, \ldots, a_{r}\right): \mathcal{D}\left(a, \ldots, a_{r-1}, \phi_{r}\right)$.

\section{Dimension, order, and degree of a proper irreducible chain}

Let $P_{1}, P_{2}$ be two r-pols and $\operatorname{lead}\left(P_{1}\right)=y_{p, q} . \quad P_{2}$ is said to be reduced w.r.t $P_{1}$ if $\operatorname{deg}\left(P_{2}, y_{p, q+i}\right)<\operatorname{deg}\left(P_{1}, y_{p, q}\right)$ for any nonnegative integer $i$.

An r-pol $P_{1}$ has higher rank than an r-pol $P_{2}$, denoted as $P_{1}>_{\text {rank }} P_{2}$, if

1). $\operatorname{class}\left(P_{1}\right)>\operatorname{class}\left(P_{2}\right)$,

2). $c=\operatorname{class}\left(P_{1}\right)=\operatorname{class}\left(P_{2}\right)$ and $\operatorname{ord}\left(P_{1}, x_{c}\right)>\operatorname{ord}\left(P_{2}, x_{c}\right)$, or

3). $\quad c=\operatorname{class}\left(P_{1}\right)=\operatorname{class}\left(P_{2}\right), o=\operatorname{ord}\left(P_{1}, x_{c}\right)=\operatorname{ord}\left(P_{2}, x_{c}\right)$ and $\operatorname{deg}\left(P_{1}, x_{c, o}\right)>$ $\operatorname{deg}\left(P_{2}, x_{c, o}\right)$.

If no one has higher rank than the other for two r-pols, they are said to have the same rank, denoted as $P_{1}={ }_{\text {rank }} P_{2}$. We use $P_{1} \leq_{\text {rank }} P_{2}$ to denote the relation of either $P_{1}<_{\text {rank }} P_{2}$ or $P_{1}={ }_{\text {rank }} P_{2}$. It is easy to see that $\leq_{\text {rank }}$ is a total order on the r-pol ring.

A finite sequence of nonzero r-pols $\mathcal{A}=A_{1}, \ldots, A_{p}$ is called an ascending chain or simply a chain, if $p=1$ and $A_{1} \neq 0$ or $\operatorname{class}\left(A_{1}\right)>0, A_{i}<_{\text {rank }} A_{j}$ and $A_{j}$ is reduced w.r.t $A_{i}$ for $1 \leq i<j \leq p$.

A chain $\mathcal{A}$ is called trivial if class $\left(A_{1}\right)=0$. For a chain (algebraic or difference), we denote $\mathbf{I}_{\mathcal{A}}$ as the product of the initials of the r-pols in $\mathcal{A}, \mathbb{I}_{\mathcal{A}}$ as the set of products of initials of the r-pols in $\mathcal{A}$ and their transforms. 
Example 3.1 Let us consider $P_{1}=\mathbf{E} y^{2}-y^{2}+1, P_{2}=\mathbf{E}^{2} y+\mathbf{E} y \in \mathcal{K}\{y\}$. Since $P_{1}<_{\text {rank }} P_{2}$, $\left.\left.\operatorname{deg}\left(P_{2}, \mathbf{E} y\right)\right)<\operatorname{deg}\left(P_{1}, \mathbf{E} y\right)\right)$ and $\operatorname{deg}\left(P_{2}, \mathbf{E}^{2} y\right)<\operatorname{deg}\left(P_{1}, \mathbf{E} y\right), P_{2}$ is reduced w.r.t $P_{1}$. Hence, $P_{1}, P_{2}$ is a chain.

Let $\mathcal{A}$ be a chain. The saturation ideal of $\mathcal{A}$ is defined as follows

$$
\operatorname{sat}(\mathcal{A})=[\mathcal{A}]: \mathbb{I}_{\mathcal{A}}=\left\{P \in \mathcal{K}\{\mathbb{X}\} \mid \exists J \in \mathbb{I}_{\mathcal{A}} \text {, s.t. } J P \in[\mathcal{A}]\right\} \text {. }
$$

If $\mathcal{B}$ is an algebraic chain, the algebraic saturation ideal of $\mathcal{B}$ is defined as follows

$$
\text { a-sat }(\mathcal{A})=\left\{P \mid \exists k \geq 0 \text {, s.t. } \mathbf{I}_{\mathcal{A}}^{k} P \in(\mathcal{A})\right\} .
$$

A chain $\mathcal{A}=A_{1}, \ldots, A_{p}$ is said to be of higher rank than another chain $\mathcal{B}=B_{1}, \ldots, B_{s}$, denoted as $\mathcal{A}>_{\text {rank }} \mathcal{B}$, if one of the following conditions holds:

1). $\exists 0<j \leq \min \{p, s\}$, such that $\forall i<j, A_{i}={ }_{\text {rank }} B_{i}$ and $A_{j}>_{\text {rank }} B_{j}$, or

2). $s>p$ and $A_{i}={ }_{\text {rank }} B_{i}$ for $i \leq p$.

If no one has higher rank than the other for two chains, they have the same rank, and is denoted as $\mathcal{A}=_{\text {rank }} \mathcal{B}$. We use $\mathcal{A}_{1} \leq_{\text {rank }} \mathcal{A}_{2}$ to denote the relation of either $\mathcal{A}_{1}<_{\text {rank }} \mathcal{A}_{2}$ or $\mathcal{A}_{1}={ }_{\text {rank }} \mathcal{A}_{2}$. It is easy to see that $\leq_{\text {rank }}$ is a total order on the difference chain set. It is known that this total order is Notherian, that is, any strictly decreasing sequence of chains must be finite.

A characteristic set of any r-pol set $\mathbb{P}$ is a chain contained in $\mathbb{P}$ and has the lowest rank. An r-pol is said to be reduced w.r.t a chain if it is reduced to every r-pol in the chain. It is known that [15].

Lemma 3.2 $\mathcal{A} \subset \mathbb{P}$ is a characteristic set of $\mathbb{P}$ if and only if there is no nonzero $r$-pol in $\mathbb{P}$ which is reduced w.r.t $\mathcal{A}$.

We can define the pseudo-remainder of an r-pol $P$ w.r.t a chain $\mathcal{A}: \operatorname{rprem}(P, \mathcal{A})$, which has the following basic property [8].

Lemma 3.3 Let $P, \mathcal{A}$ be as above, and $R=\operatorname{rprem}(P, \mathcal{A})$. Then there is a $J \in \mathbb{I}_{\mathcal{A}}$ with $\operatorname{lead}(J)<_{\text {rank }}$ lead $(P)$ such that $J P \equiv R \bmod [\mathcal{A}]$ and $R$ is reduced w.r.t $\mathcal{A}$.

For any chain $\mathcal{A}$, after a proper renaming of the variables, we could write it as the following form.

$$
\mathcal{A}=\left\{\begin{array}{l}
A_{1,1}\left(\mathbb{U}, y_{1}\right), \ldots, A_{1, k_{1}}\left(\mathbb{U}, y_{1}\right) \\
\ldots \\
A_{p, 1}\left(\mathbb{U}, y_{1}, \ldots, y_{p}\right), \ldots, A_{p, k_{p}}\left(\mathbb{U}, y_{1}, \ldots, y_{p}\right)
\end{array}\right.
$$

where $\operatorname{lvar}\left(A_{i, j}\right)=y_{i}$ and $\mathbb{U}=\left\{u_{1}, \ldots, u_{q}\right\}$ such that $p+q=n$. Let $o_{(i, j)}=\operatorname{ord}\left(A_{i, j}, y_{i}\right) . \mathbb{U}$ is called the parametric set of $\mathcal{A}$. We define the dimension of $\mathcal{A}$ as $\operatorname{dim}(\mathcal{A})=|\mathbb{U}|$, the degree of $\mathcal{A}$ as $\operatorname{deg}(\mathcal{A})=\prod_{i=1}^{p} \operatorname{deg}\left(A_{i, k_{i}}, y_{i, o_{\left(i, k_{i}\right)}}\right)$, the order of $\mathcal{A}$ as $\operatorname{ord}(\mathcal{A})=\sum_{i=1}^{p} o_{(i, 1)}$, and the effective order of $\mathcal{A}$ as $\operatorname{Eord}(\mathcal{A})=\sum_{i=1}^{p}\left(o_{(i, 1)}-m_{i}\right)$ where $m_{i}$ is the minimal integer such that $y_{i, m_{i}}$ occurs in $A_{i, 1}$.

Let $h_{1}, \ldots, h_{m}(m \leq p)$ be nonnegative integers. We use $\mathcal{A}_{\left(h_{1}, \ldots, h_{m}\right)}$ to denote the following sequence of r-pols

$$
\begin{aligned}
& A_{1,1}, \mathbf{E} A_{1,1}, \ldots, \mathbf{E}^{o_{(1,2)}-o_{(1,1)}-1} A_{1,1}, A_{1,2}, \ldots, \quad A_{1, k_{1}}, \mathbf{E} A_{1, k_{1}}, \ldots, \mathbf{E}^{\hat{h}_{1}-o_{\left(1, k_{1}\right)}} A_{1, k_{1}}, \\
& \ldots, \\
& A_{m, 1}, \mathbf{E} A_{m, 1}, \ldots, \mathbf{E}^{o_{(m, 2)}-o_{(m, 1)}-1} A_{m, 1}, A_{m, 2}, \ldots, \quad A_{m, k_{m}}, \mathbf{E} A_{m, k_{m}}, \ldots, \mathbf{E}^{\hat{h}_{m}-o_{\left(m, k_{m}\right)}} A_{m, k_{m}}
\end{aligned}
$$


where $\hat{h}_{i}$ is defined as follows: $\hat{h}_{m}=\max \left\{h_{m}, o_{\left(m, k_{m}\right)}\right\}+1$, and for $i=m-1, \ldots, 1, o_{i}$ $=\max \left\{\right.$ order of $y_{i}(x)$ appears in $\left.A_{i+1,1}, \mathbf{E} A_{i+1,1}, \ldots, \mathbf{E}^{\hat{h}_{m}-o_{\left(m, k_{m}\right)}} A_{m, k_{m}}\right\}, \hat{h}_{i}=\max \left\{h_{i}, o_{i}, o_{\left(i, k_{i}\right)}\right\}$ +1 . It is obvious that $\mathcal{A}_{\left(h_{1}, \ldots, h_{m}\right)}$ is an algebraic triangular set with parameters:

$$
\mathcal{P}(\mathcal{A})=\left\{y_{i, j} \mid 1 \leq i \leq p, 0 \leq j \leq \operatorname{ord}\left(A_{i, 1}, y_{i}\right)-1\right\} .
$$

For a chain $\mathcal{A}$ and an r-pol $P$, let

$$
\begin{aligned}
\mathcal{A}^{*} & =\mathcal{A}_{(0, \ldots, 0)} \\
\mathcal{A}_{P} & =\mathcal{A}_{\left(\operatorname{ord}\left(P, y_{1}\right), \ldots, \operatorname{ord}\left(P, y_{p}\right)\right)}
\end{aligned}
$$

Let $\mathcal{A}=A_{1}, \ldots, A_{p}$ be an algebraic nontrivial triangular set in $\mathcal{K}\left[x_{1}, \ldots, x_{n}\right]$ over a field $\mathcal{K}$. Let $y_{i}$ be the leading variable of $A_{i}, Y=\left\{y_{1}, \ldots, y_{p}\right\}$ and $U$ the parametric set of $\mathcal{A}$. A polynomial $f$ is said to be invertible w.r.t $\mathcal{A}$ if either $f \in \mathcal{K}[U]$ or $\left(f, A_{1}, \ldots, A_{s}\right) \cap \mathcal{K}[U] \neq\{0\}$ where $y_{s}$ is the leading variable of $f$. An r-pol $P$ is said to be invertible w.r.t $\mathcal{A}$ if it is invertible w.r.t $\mathcal{A}_{P}$ when $P$ and $\mathcal{A}_{P}$ are treated as algebraic polynomials.

An r-pol $P$ is called effective in variable $y_{i}$ if $y_{i, 0}=y_{i}(x)$ occurs in $P$. $P$ is called effective if $P$ is effective in its leading variable.

Let $\mathcal{A}=A_{1}, \ldots, A_{m}$ be a difference chain in $\mathcal{K}\{\mathbb{X}\}$ and $k_{i}=\operatorname{ord}\left(A_{i}, \operatorname{lvar}\left(A_{i}\right)\right), i=$ $1, \ldots, m$. For any $1 \leq i<j \leq m$, if $\operatorname{class}\left(A_{i}\right)=\operatorname{class}\left(A_{j}\right)=t$, let $\Delta_{i j}=\operatorname{prem}\left(\mathbf{E}^{k_{j}-k_{i}} A_{i}\right.$, $\left.A_{j}, y_{t, k_{j}}\right)$ be the algebraic pseudo-remainder of $\mathbf{E}^{k_{j}-k_{i}} A_{i}$ w.r.t $A_{j}$ in variable $y_{t, k_{j}}$; otherwise, let $\Delta_{i j}=0$. If $\operatorname{rprem}\left(\Delta_{i j}, \mathcal{A}\right)=\operatorname{prem}\left(\Delta_{i j}, \mathcal{A}^{*}\right)=0$, we call $\mathcal{A}$ a coherent difference chain.

Definition 3.4 A chain $\mathcal{A}$ of the form (1) is said to be proper irreducible if

- $\mathcal{A}^{*}$ as defined in (4) is an algebraic irreducible chain; and

- For $c=1, \ldots, p, A_{c, 1}$ is effective and $\hat{A}_{c, 1}$ is irreducible in $\mathcal{K}\left(\eta_{c-1}\right)\left[y_{c}(x), \ldots, y_{c}\left(x+f_{c}\right)\right]$, where $f_{c}=\operatorname{ord}\left(A_{c, 1}, y_{c}\right), \mathcal{B}_{c}=\mathcal{A}^{*} \cap \mathcal{K}\left\{\mathbb{U}, y_{1}, \ldots, y_{c}\right\} \quad\left(\mathcal{B}_{0}=\emptyset\right), \eta_{c}$ is a generic point for the algebraic irreducible chain $\mathcal{B}_{c}$, and $\hat{A}_{c, 1}$ is obtained by substituting $\eta_{c-1}$ into $A_{c, 1}$.

Proper irreducible chains have the following basic properties[8].

Lemma 3.5 Let $\mathcal{A}$ be a coherent and proper irreducible chain of the form (1). If $P$ is invertible w.r.t $\mathcal{A}$, then $\mathbf{E} P$ is invertible w.r.t $\mathcal{A}$.

Lemma 3.6 Let $\mathcal{A}$ be a coherent and proper irreducible chain. Then $\mathcal{A}$ is a characteristic set of $\operatorname{sat}(\mathcal{A})$.

Lemma 3.7 Let $\mathcal{A}$ be a coherent and proper irreducible chain of the form (1), and $f \in$ $\mathcal{K}\{\mathbb{U}, Y\}, g \in \mathcal{K}\{\mathbb{U}\}[\mathcal{P}(\mathcal{A})] \backslash\{0\}$. If $g f \in \operatorname{sat}(\mathcal{A})$, then $f \in \operatorname{sat}(\mathcal{A})$.

Proof: Let $\operatorname{rprem}(f, \mathcal{A})=R$. Then there is a $J \in \mathbb{I}_{\mathcal{A}}$ such that

$$
J f=R \bmod [\mathcal{A}] .
$$

Therefore, $g R=J g f \bmod [\mathcal{A}]$. Since $g f \in \operatorname{sat}(\mathcal{A}), g R \in \operatorname{sat}(\mathcal{A})$. Since $\mathcal{A}$ is coherent and proper irreducible, by Lemma 3.6, $\mathcal{A}$ is the characteristic set of $\operatorname{sat}(\mathcal{A})$. Since $g R$ is reduced w.r.t $\mathcal{A}$, we have $g R=0$, and hence $R=0$. 
Definition 3.8 A proper irreducible chain $\mathcal{A}$ of the form (1) is said to be strong irreducible if for any nonnegative integers $h_{i}, \mathcal{A}_{\left(h_{1}, \ldots, h_{p}\right)}$ is an irreducible triangular set in algebraic case.

It is proved that if $\mathcal{A}$ is a coherent and strong irreducible chain, then $\operatorname{sat}(\mathcal{A})$ is a reflexive prime ideal [8].

Theorem 3.9 Let $\mathcal{A}$ be a coherent and proper irreducible chain of the form (1). We have:

(1) $\mathbb{U}$ is a parametric set of ideal $\operatorname{sat}(\mathcal{A})$. That is, $\operatorname{sat}(\mathcal{A}) \cap \mathcal{K}\{\mathbb{U}\}=\{0\}$ and $\forall i$, $\operatorname{sat}(\mathcal{A}) \cap$ $\mathcal{K}\left\{\mathbb{U}, y_{i}\right\} \neq\{0\}$.

(2) If $\{\operatorname{sat}(\mathcal{A})\}=\bigcap_{i=1}^{r} P_{i}$ is an irredundant intersection of a set of prime difference ideals, then $\forall i, \mathbb{U}$ is a parametric set for $P_{i}$ and thus $\operatorname{dim}\left(P_{i}\right)=|\mathbb{U}|$.

(3) $\operatorname{Eord}_{\mathbb{U}} P_{i}=\operatorname{ord}_{\mathbb{U}} P_{i}=\operatorname{ord}(\mathcal{A})$ and $P_{i}$ is reflexive. Let $P_{i}=\operatorname{sat}\left(\mathcal{A}_{i}\right)$, and $A_{i}$ a chain under the same variable order as $\mathcal{A}$. Then $\mathcal{A}_{i}$ is strong irreducible.

(4) $\operatorname{deg}(\mathcal{A})=\sum_{i=1}^{r} \operatorname{ld}_{\mathbb{U}}\left(P_{i}\right)$.

Proof: By Lemma 3.6, $\mathcal{A}$ is a characteristic set of $\operatorname{sat}(\mathcal{A})$. As a consequence, we have $\operatorname{sat}(\mathcal{A}) \cap \mathcal{K}\{\mathbb{U}\}=\emptyset$, since every non-zero r-pol in $\operatorname{sat}(\mathcal{A}) \cap \mathcal{K}\{\mathbb{U}\}$ is reduced w.r.t to $\mathcal{A}$ and hence must be zero. If there exists an $i$, such that $\operatorname{sat}(\mathcal{A}) \cap \mathcal{K}\left\{\mathbb{U}, y_{i}\right\}=\emptyset$, let $h=|\mathcal{P}(\mathcal{A})|$ and $\mathcal{C}=\mathcal{A}_{(0, \ldots, 0, h, 0, \ldots, 0)}$, where $h$ is at the $i$-th place. Let $Y^{\prime}$ be the set of all $y_{i, j}$ occurring in $\mathcal{C}$, and $k$ large enough such that all the $u_{i, j}$ occurring in $\mathcal{C}$ satisfy $j \leq k$. Let $\mathbb{U}_{k}=$ $\left\{u_{i, j}, 0 \leq i \leq 1,0 \leq j \leq k\right\}$. Since all the initials of $\mathcal{C}$ are invertible w.r.t $\mathcal{C}$, Zero $(\operatorname{sat}(\mathcal{C}))$ is an unmixed algebraic ideal of $\operatorname{dimension} \operatorname{dim}(A)=h$ in $\mathcal{K}\left(\mathbb{U}_{k}\right)\left[Y^{\prime}\right][6]$. On the other hand, $\operatorname{sat}(\mathcal{C}) \cap \mathcal{K}\left(\mathbb{U}_{k}\right)\left[y_{i, 0}, \ldots, y_{i, h}\right] \subset \operatorname{sat}(\mathcal{A}) \cap \mathcal{K}\left(\mathbb{U}_{k}\right)\left[y_{i, 0}, \ldots, y_{i, h}\right]=\{0\}$. From this, we have $\operatorname{dim}(\mathbf{s a t}(\mathcal{C})) \geq h+1$, a contradiction. This proves $(1)$.

Let $\{\operatorname{sat}(\mathcal{A})\}=\bigcap_{i=1}^{r} P_{i}$ be an irredundant intersection of a set of prime difference ideals. Since $\forall i,\{\operatorname{sat}(\mathcal{A})\} \subseteq P_{i}$, for each $i$ we have $P_{i} \cap \mathcal{K}\left\{\mathbb{U}, y_{i}\right\} \neq\{0\}$. Now we need only to prove that $\mathbb{U}$ is also an transform independent set of $P_{i}$. Suppose that there exists an $i$, say $i=1$, such that $P_{1} \cap \mathcal{K}\{\mathbb{U}\} \neq\{0\}$. Then $\exists g \in \mathcal{K}\{\mathbb{U}\} \cap P_{i}$ and $g \neq 0$. Since $\{\operatorname{sat}(\mathcal{A})\}=\bigcap_{i=1}^{r} P_{i}$ is an irredundant representation, there exists an $f \in \mathcal{K}\{\mathbb{U}, Y\}$, such that $f \in \bigcap_{i=2}^{r} P_{i}, f \notin\{\operatorname{sat}(\mathcal{A})\}$. We have $g f \in\{\operatorname{sat}(\mathcal{A})\}$. So there exist non negative integers $s_{0}, s_{1}, \ldots, s_{r}$, such that

$$
\Pi_{i=0}^{r}\left(\mathbf{E}^{i}(g f)\right)^{s_{i}}=\Pi_{i=0}^{r}\left(\mathbf{E}^{i}(g)\right)^{s_{i}} * \Pi_{i=0}^{r}\left(\mathbf{E}^{i}(f)\right)^{s_{i}} \in \mathbf{s a t}(\mathcal{A}) .
$$

By Lemma 3.7, we have $\prod_{i=0}^{r}\left(\mathbf{E}^{i}(f)\right)^{s_{i}} \in \mathbf{s a t}(\mathcal{A})$. Therefore, $f \in\{\operatorname{sat}(\mathcal{A})\}$, which contradicts to the choice of $f$. So $\mathbb{U}$ is a transform independent set of $P_{i}$. Hence $\operatorname{dim}\left(P_{i}\right)=|\mathbb{U}|$. This proves (2).

Let $o_{i}=\operatorname{ord}\left(A_{i, 1}, y_{i}\right)$. It is clear that $|\mathcal{P}(\mathcal{A})|=\sum_{i=1}^{p} o_{i}$. Since $\mathcal{A}$ is proper irreducible, for any $y_{i j}, j \geq o_{i}$, there is a nonzero $P \in \operatorname{sat}(\mathcal{A})$ such that $P \in \mathcal{K}\{\mathbb{U}\}\left[\mathcal{P}(\mathcal{A}), y_{i, j}\right]$. Since $\operatorname{sat}(\mathcal{A}) \subseteq P_{i}$, so $\operatorname{ord}_{\mathbb{U}} P_{i} \leq=\sum_{i=1}^{p} o_{i}$. If there exists an $i$, say $i=1$, such that $P_{i} \cap \mathcal{K}\{\mathbb{U}\}[\mathcal{P}(\mathcal{A})] \neq\{0\}$, then there exists a $g \neq 0, g \in P_{1} \cap \mathcal{K}\{\mathbb{U}\}[\mathcal{P}(\mathcal{A})]$. Also, there exists 
an $f \in \mathcal{K}\{\mathbb{U}, Y\}$, such that $f \in \bigcap_{i=2}^{r} P_{i}, f \notin\{\operatorname{sat}(\mathcal{A})\}$ and $g f \in\{\operatorname{sat}(\mathcal{A})\}$. There exist non negative integers $s_{0}, \ldots, s_{l}$ such that

$$
\Pi_{i=0}^{l}\left(\mathbf{E}^{i}(g f)\right)^{s_{i}} \in \mathbf{s a t}(\mathcal{A}) .
$$

Since $g$ is invertible w.r.t $\mathcal{A}$, by Lemma $3.5, \mathbf{E}^{i} g$ is also invertible w.r.t $\mathcal{A}$. So, there exists $g^{\prime} \in$ $\mathcal{K}\{\mathbb{U}, Y\}$, such that $g^{\prime} * \Pi_{i=0}^{l}\left(\mathbf{E}^{i} g\right)^{s_{i}}=M \neq 0, M \in K\{\mathbb{U}\}[\mathcal{P}(\mathcal{A})]$. Then $M * \Pi_{i=0}^{l}\left(\mathbf{E}^{i} f\right)^{s_{i}} \in$ $\operatorname{sat}(\mathcal{A})$. By Lemma 3.7, $\Pi_{i=0}^{l}\left(\mathbf{E}^{i} f\right)^{s_{i}} \in \mathbf{s a t}(\mathcal{A})$, so $f \in\{\mathbf{s a t}(\mathcal{A})\}$, a contradiction. Therefore, $\mathbb{U} \cup \mathcal{P}(\mathcal{A})$ is algebraic independent in $P_{i}$ and hence $\operatorname{ord}_{\mathbb{U}} P_{i} \geq|\mathcal{P}(\mathcal{A})|$. Then $\operatorname{ord}_{\mathbb{U}} P_{i}=\operatorname{ord}(\mathcal{A})$. Let $\mathcal{P}(\mathcal{A})^{(T)}=\left\{E^{T_{i}} y_{i j} \mid y_{i j} \in \mathcal{P}(\mathcal{A})\right\}$, where $T=\left(T_{1}, \ldots, T_{p}\right)$ is a set of non negative integers. Notice that $\{\operatorname{sat}(\mathcal{A})\}=\bigcap_{i=1}^{r} P_{i}$ is an irredundant representation and $\mathcal{A}$ is effective. In each $P_{i}$, using the properties of the transcendental degree, we have $P_{i} \cap \mathcal{K}\langle\mathbb{U}\rangle\left[\mathcal{P}(\mathcal{A})^{(T)}\right]=\{0\}$. So $\operatorname{Eord}_{\mathbb{U}} P_{i} \geq|\mathcal{P}(\mathcal{A})|$. Since $\operatorname{Eord}_{\mathbb{U}} P_{i} \leq \operatorname{ord}_{\mathbb{U}} P_{i}=|\mathcal{P}(\mathcal{A})|$, we have $\operatorname{Eord}_{\mathbb{U}} P_{i}=\operatorname{ord}_{\mathbb{U}} P_{i}$. By Lemma $2.2, P_{i}$ is reflexive.

In order to prove $A_{i}$ is strong irreducible, we need only to prove that $\mathcal{A}_{i}$ is effective. Let $A_{i, j}$ be the first r-pol in $\mathcal{A}_{i}$ with $\operatorname{lvar}\left(A_{i, j}\right)=y_{j}$. We need only to prove that $A_{i, j}$ is effective in $y_{j}$. In the above, we already proved that $\operatorname{ord}\left(A_{i, j}, y_{j}\right)=o_{j}$. If $A_{i, j}$ is not effective, it does not involve $y_{j, 0}$ Let $\mathcal{P}_{j-1}=\left\{y_{i, k} \mid 1 \leq i \leq j-1,0 \leq k \leq o_{i}-1\right\}$. By the properties of algebraic independence, from $A_{i, j}$, we may obtain an r-pol $Q \in \mathcal{K}\left[\mathbb{U}, \mathcal{P}_{j-1}, y_{j, 1}, \ldots, y_{j, o_{j}}\right]$ such that $Q \in P_{i}$. This is impossible, because from $P_{i} \cap \mathcal{K}\left[\mathbb{U}, \mathcal{P}_{j-1}, y_{j, 0}, \ldots, y_{j, o_{j}-1}\right]=\{0\}$ and $\operatorname{Eord}_{\mathbb{U}} P_{i}=\operatorname{ord}_{\mathbb{U}} P_{i}$, we have $P_{i} \cap \mathcal{K}\left[\mathbb{U}, \mathcal{P}_{j-1}, y_{j, 1}, \ldots, y_{j, o_{j}}\right]=\{0\}$. We now proved (3).

We denote a generic zero of $\mathcal{A}^{*}$ as $\eta=\left(\alpha_{i j}, \beta_{i j}\right)$, where $\alpha_{i j}, \beta_{i j}$ correspond to $u_{i j}, y_{i j}$ respectively. By the proof of Theorem 4.2 in [8], we know that there exists a difference kernel $R$ of length one: $\mathbf{E}: \mathcal{K}\left(a_{0}\right) \rightarrow \mathcal{K}\left(a_{1}\right)$, and $\alpha=\left\{\alpha_{i j} \mid 1 \leq i \leq q, j \geq 0\right\} \cap a_{1}$ is a parametric set of $R, \operatorname{deg}_{\mathbb{U}} R=\mathcal{K}\left(a_{1}\right): \mathcal{K}\left(a_{0}, \alpha\right)=\operatorname{deg}(\mathcal{A})$. By Lemma 5 in chapter 6 of [2], we know that $R$ has a finite number of principal realizations, denoted as $V_{i}, 1 \leq i \leq m$, and its generic zero denoted as $\eta_{i}$. We define $\operatorname{Spec}\left(V_{i}\right)=\left\{f \in \mathcal{K}\{\mathbb{U}, \mathbb{Y}\} \mid f\left(\eta_{i}\right)=0\right\}$, $\operatorname{son} \operatorname{sat}(\mathcal{A}) \subset \operatorname{Spec}\left(V_{i}\right)$ and $\operatorname{Spec}\left(V_{i}\right)$ is a prime difference ideal. Since $\operatorname{sat}(\mathcal{A})=\bigcap_{i=1}^{r} P_{i} \subset \operatorname{Spec}\left(V_{i}\right)$, there exists a $j$ such that $\operatorname{Spec}\left(V_{i}\right) \subset P_{j}$. Since $P_{j}$ and $\operatorname{Spec}\left(V_{i}\right)$ have the same dimension and order, $\operatorname{Spec}\left(V_{i}\right)=P_{j}$. So for each $i$, there exists an $l_{i}$, such that $\operatorname{Spec}\left(V_{i}\right)=P_{l_{i}}$ and for each $P_{j}$, its generic zero is a principal realization of $R$, so it must be some $\eta_{i}$. Hence, there is a one to one corresponding between $V_{i}$ and $P_{j}$. By Lemma 5 in chapter 6 of [2], we have $\operatorname{deg}(\mathcal{A})=\operatorname{deg}_{\mathbb{U}} R=\sum_{i=1}^{m} \operatorname{ld}_{\mathbb{U}} V_{i}=\sum_{i=1}^{r} \operatorname{ld}_{\mathbb{U}}\left(P_{i}\right)$. This proves $(4)$. I

If two ideals $I_{1}$ and $I_{2}$ have the same parametric set $\mathbb{U}$ and $\operatorname{ord}_{\mathbb{U}} I_{1}=\operatorname{ord}_{\mathbb{U}} I_{2}$ and $\operatorname{Eord}_{\mathbb{U}} I_{1}=\operatorname{Eord}_{\mathbb{U}} I_{2}$, then we say that $I_{1}$ and $I_{2}$ are of the same type. As a consequence of Theorem 3.9, we have the following corollaries (proofs omitted).

Corollary 3.10 If $\mathcal{A}$ is coherent and proper irreducible, all the irredundant prime divisors of $\operatorname{sat}(\mathcal{A})$ are of the the same type with $\operatorname{sat}(\mathcal{A})$. In other words, $\operatorname{sat}(\mathcal{A})$ is unmixed in the sense that all its essential prime ideals have the same parametric set, the same dimension, and the same order w.r.t the parametric set.

As a consequence, we can define $\operatorname{dim}(\operatorname{sat}(\mathcal{A}))=\operatorname{dim}(\mathcal{A})$ and $\operatorname{ord} \mathbb{U}(\operatorname{sat}(\mathcal{A}))=\operatorname{ord}(\mathcal{A})$.

It is proved in [8] that if $\mathcal{A}$ is coherent and strong irreducible, then $\operatorname{sat}(\mathcal{A})$ is a reflexive prime ideal with $\mathbb{U}$ as a set of parameters. Furthermore, we have 
Corollary 3.11 If $\mathcal{A}$ is a coherent and strong irreducible chain, then $\operatorname{sat}(\mathcal{A})$ is a reflexible prime ideal of dimension $\operatorname{dim}(\mathcal{A})$, order $\operatorname{ord}(\mathcal{A})$ w.r.t $\mathbb{U}$, and limit degree $\operatorname{deg}(\mathcal{A})$ w.r.t $\mathbb{U}$.

Corollary 3.12 Let $\mathcal{A}$ be a coherent and proper irreducible chain, and

$$
\{\operatorname{sat}(\mathcal{A})\}=\bigcap_{i=1}^{r} \operatorname{sat}\left(\mathcal{A}_{i}\right)
$$

a decomposition of $\{\boldsymbol{\operatorname { s a t }}(\mathcal{A})\}$ as irredundant prime difference ideals, where $\mathcal{A}_{i}$ are coherent and strong irreducible chains under the same variable order as that of $\mathcal{A}$. Then the first r-pol of $\mathcal{A}_{i}$ is the same as the first r-pol of $\mathcal{A}$.

Proof: Let $f$ and $f_{i}$ be the first element of $\mathcal{A}$ and $\mathcal{A}_{i}$ respectively. Then $f$ and $f_{i}$ are irreducible and of the same order $o$ w.r.t $y_{1}$. Since $f \in \operatorname{sat}\left(\mathcal{A}_{i}\right)$ and $\mathcal{A}_{i}$ is a characteristic set of $\mathcal{A}_{i}$, we have $\operatorname{rprem}\left(f, \mathcal{A}_{i}\right)=\operatorname{prem}\left(f, f_{i}\right)=0$. Since $f$ and $f_{i}$ are irreducible and of the same lead, we have $f=f_{i}$. I

Corollary 3.13 Let $\mathcal{A}$ be a coherent and proper irreducible chain of the form (1). If $\{\operatorname{sat}(\mathcal{A})\}$ has only one prime component, then $\mathcal{A}$ is strong irreducible and $\operatorname{sat}(\mathcal{A})$ is a prime ideal.

Proof: Let $\mathcal{B}$ be a characteristic set of the prime component of $\{\operatorname{sat}(\mathcal{A})\}$ under the same variable order. Then by Theorem 3.9,

$$
\{\operatorname{sat}(\mathcal{A})\}=\operatorname{sat}(\mathcal{B})
$$

By (4) of Theorem 4.1 and Corollary 3.11, we have $\operatorname{deg}(\mathcal{A})=\operatorname{deg}(\mathcal{B})$. This means that $\mathcal{A}_{\left(h_{1}, \ldots, h_{p}\right)}$ must be an irreducible algebraic triangular set for all non-negative integers $h_{i}$. Otherwise, the limit degree of $\{\operatorname{sat}(\mathcal{A})\}$ w.r.t $\mathbb{U}$ will be less than $\operatorname{deg}(\mathcal{D})$, a contradiction. I

\section{Resolvent system for a reflexive prime ideal}

It is proved in [8] that for a reflexive prime difference ideal $I$, we can choose a proper order of variables such that under this variable order $I=\operatorname{sat}(\mathcal{A})$ and $\mathcal{A}$ is coherent and strong irreducible. So, we will start our discussion from a coherent and strong irreducible chain.

\subsection{Resolvent systems for a reflexive prime ideal}

Theorem 4.1 Let $\mathcal{A}$ be a coherent and strong irreducible chain of the form (1), $\mathcal{K}$ an aperiodic difference field or $|\mathbb{U}| \neq 0$, and $\lambda_{1}, \ldots, \lambda_{p}$ difference variables. There exists $Q \in$ $\mathcal{K}\left\{\lambda_{1}, \ldots, \lambda_{p}, \mathbb{U}\right\}$ such that if $\sigma_{1}, \ldots, \sigma_{p}$ satisfy $Q\left(\sigma_{1}, \ldots, \sigma_{p}, \mathbb{U}\right) \neq 0$, then the characteristic set of $\operatorname{sat}\left(\mathcal{A}, w-\sum_{i=1}^{p} \sigma_{i} y_{i}\right)$ under the variable order $\mathbb{U}<w<y_{i}$ is of the following form

$$
R, R_{1}, \ldots, R_{s}, I_{1} y_{1,0}-V_{1}, \ldots, I_{p} y_{p, 0}-V_{p}
$$

where $R, R_{i}, I_{i}, V_{i} \in K\{\mathbb{U}, w\}$. Furthermore, $R$ is effective in $w, \operatorname{ord}(R, w)=\operatorname{ord}(\mathcal{A})$, $\operatorname{ord}\left(I_{i}, w\right)<\operatorname{ord}(\mathcal{A})$, and $\operatorname{ord}\left(V_{p}, w\right) \leq \operatorname{ord}(\mathcal{A})$. 
Proof: Denote $\mathcal{B}=\mathcal{A}, w-\sum_{i=1}^{p} \lambda_{i} y_{i}$. Then $\mathcal{B}$ is also a coherent and strong irreducible chain and $\operatorname{sat}(\mathcal{B})=\left\{\operatorname{sat}(\mathcal{A}), w-\sum_{i=1}^{p} \lambda_{i} y_{i}\right\}$ is also a reflexive prime ideal. Since $\mathbb{U}$ is a parametric set of $\operatorname{sat}(\mathcal{B})$, we could treat $\mathcal{K}_{0}=\mathcal{K}\langle\mathbb{U}, \lambda\rangle$ as the ground field where $\lambda=$ $\left\{\lambda_{1}, \ldots, \lambda_{p}\right\}$. We denote $\operatorname{ord}_{X}(Z)$ as the largest number of elements of the subsets of $Z$ and their transformations, such that it is algebraic independence over $\mathcal{K}_{0}\langle X\rangle$. Let $\left(\alpha_{1}, \ldots, \alpha_{p}, \omega\right)$ be a generic zero of $\operatorname{sat}(\mathcal{B})$, and $\alpha=\left\{\alpha_{1}, \ldots, \alpha_{p}\right\}$. Then

$$
\begin{aligned}
\operatorname{Eord}(\operatorname{sat}(\mathcal{A})) & =\operatorname{Eord}(\operatorname{sat}(\mathcal{B}))=\operatorname{Eord}(\omega)+\operatorname{Eord}_{\omega}(\alpha) \\
& \leq \operatorname{ord}(\omega)+\operatorname{ord}_{\omega}(\alpha)=\operatorname{ord}(\omega, \alpha) \\
& =\operatorname{ord}(\operatorname{sat}(\mathcal{A}))=\operatorname{Eord}(\operatorname{sat}(\mathcal{A})) .
\end{aligned}
$$

The last equation is due to Corollary 3.11. As a consequence, $\operatorname{Eord}(\omega)=\operatorname{ord}(\omega)$. Let $T(w) \in$ $\operatorname{sat}(\mathcal{B})$ be an r-pol in $\mathbb{U}$ and $w$ with the lowest rank. Since $\operatorname{sat}(\mathcal{B})$ is a prime ideal, $T(w)$ can be chosen as an irreducible r-pol. Also, $T(w)$ must be the first element of the characteristic set of $\operatorname{sat}(\mathcal{B})$ under the variable order $\mathbb{U}<\lambda_{i}<w<y_{i}$. Since $\operatorname{Eord}(\omega)=\operatorname{ord}(\omega)$, by Corollary 3.11, we have $\operatorname{Eord}(T, w)=\operatorname{ord}(T, w) \leq o_{\mathcal{A}}$. In other words, $T$ is effective in $w$. Since $\lambda_{i}$ are transformally transcendental over $\mathcal{K}\langle\mathbb{U}\rangle$, so the coefficients of the power products of the $\lambda_{i 0}$ in the expression obtained by replacing each $\left(y_{1}, \ldots, y_{p}\right)$ by $\alpha=\left(\alpha_{1}, \ldots, \alpha_{p}\right)$, and replace $w$ by $\sum_{i=1}^{p} \lambda_{i} \alpha_{i}$ formally are all zero. Differentiating $T$ w.r.t $\lambda_{i 0}$ and substituting $\alpha_{i}$ for $y_{i}$, we have

$$
\frac{\partial T}{\partial \lambda_{i 0}}+\left.y_{i, 0} \frac{\partial T}{\partial w_{0}}\right|_{\left(y_{1}, \ldots, y_{p}, w\right)=\left(\alpha_{1}, \ldots, \alpha_{p}, \sum_{i=1}^{p} \lambda_{i} \alpha_{i}\right)}=0
$$

Since $(\alpha, \omega)$ is a generic zero of $\operatorname{sat}(\mathcal{B}), S_{i}=\frac{\partial T}{\partial \lambda_{i 0}}+y_{i 0} \frac{\partial T}{\partial w_{0}} \in \operatorname{sat}(\mathcal{B}), 1 \leq i \leq p$. Let $H$ be the resultant of $\frac{\partial T}{\partial w_{0}}$ and $T$ w.r.t $w_{o}$. We have $\operatorname{ord}(H, w)<o$. Then there exist r-pols $A, B$ such that $A T+B \frac{\partial T}{\partial w_{0}}=H$. Let $Q_{i}=B S_{i}+A T y_{i, 0}=H y_{i, 0}+B \frac{\partial T}{\partial \lambda_{i 0}} \in \operatorname{sat}(\mathcal{B})$. Let $P_{i}=$ $\operatorname{rprem}\left(Q_{i}, T, w_{o}\right)=I_{i 0} y_{i 0}+V_{i 0}$. Then $I_{i 0} \neq 0$ and $P_{i} \in \operatorname{sat}(\mathcal{B})$, where $I_{0}, V_{i 0} \in \mathcal{K}\{\mathbb{U}, \lambda, w\}$, $\operatorname{ord}\left(I_{i 0}, w\right)<\operatorname{ord}(\mathcal{A})$, and $\operatorname{ord}\left(V_{i 0}, w\right) \leq \operatorname{ord}(\mathcal{A})$. Since $T$ is irreducible and has the lowest order in $w$, we have $I_{i 0} \notin \operatorname{sat}(\mathcal{B})$. From $P_{i} \in \operatorname{sat}(\mathcal{B})$, we have $\operatorname{Eord}_{w}\left(y_{i}\right)=\operatorname{ord}_{w}\left(y_{i}\right)=0$. By (6), we have

$$
\operatorname{Eord}(T, w)=\operatorname{ord}(T, w)=\operatorname{ord}(\mathcal{A}) .
$$

Since $T, P_{i} \in \operatorname{sat}(\mathcal{B})$, the characteristic set of $\operatorname{sat}(\mathcal{B})$ under the variable order $\mathbb{U}<\lambda_{i}<w<$ $y_{i}$ must be of the form:

$$
T, T_{1}, \ldots, T_{s}, P_{1}, \ldots, P_{p}
$$

where $T_{i} \in \mathcal{K}\{\mathbb{U}, \lambda, w\}$. Let $Q \in \mathcal{K}\{\mathbb{U}, \lambda\}$ be the product of all the coefficients of $\operatorname{rprem}\left(I_{i 0}, \mathcal{B}\right)$ as a polynomial in $y_{i, j}$. Since $\mathcal{K}\langle\mathbb{U}\rangle$ is aperiodic, there exist $\sigma_{1}, \ldots, \sigma_{p} \in \mathcal{K}\{\mathbb{U}\}$ such that $Q\left(\sigma_{1}, \ldots, \sigma_{p}\right) \neq 0$. Let $\overline{\mathcal{B}}$ be the chain $\mathcal{A}, w-\sum_{i=1}^{p} \sigma_{i} y_{i}$. We have $\bar{I}_{0} y_{i 0}+\bar{V}_{i 0} \in \operatorname{sat}(\overline{\mathcal{B}})$, where $\bar{I}_{0}, \bar{V}_{i 0}$ are obtained by replacing each $\lambda_{i}$ with $\sigma_{i}$ in $Q, V_{i 0}$ respectively. Since $Q\left(\sigma_{1}, \ldots, \sigma_{p}\right) \neq$ 0 , we have $\bar{I}_{0} \notin \operatorname{sat}(\overline{\mathcal{B}})$. We denote $I_{i}=\bar{I}_{0}, V_{i}=\bar{V}_{i 0}$. Then $\bar{P}_{i}=I_{i} y_{i, 0}+V_{i} \in \operatorname{sat}(\overline{\mathcal{B}})$. From $\operatorname{ord}\left(I_{0}, w\right)<\operatorname{ord}(\mathcal{A})$ and $\operatorname{ord}\left(V_{i 0}, w\right) \leq \operatorname{ord}(\mathcal{A})$, we have $\operatorname{ord}\left(I_{i}, w\right)<\operatorname{ord}(\mathcal{A})$ and $\operatorname{ord}\left(V_{i}, w\right) \leq$ $\operatorname{ord}(\mathcal{A})$. As a consequence, a characteristic set of $\operatorname{sat}(\overline{\mathcal{B}})$ must be of the form $(5)$. Repeat the process for proving equation (6), we can show that $\operatorname{Eord}(R, w)=\operatorname{ord}(R, w)=\operatorname{ord}(\mathcal{A})$. Hence $R$ is effective in $w$.

Now, we may extend the following well-known result in algebraic geometry to difference case. 
Corollary 4.2 Any irreducible difference variety $V$ is birationally equivalent to an irreducible difference variety of codimension one if the ground field $\mathcal{K}$ is aperiodic or $V$ is of positive dimension.

Proof: Let $I$ be the set of r-pols which vanish on $V$. Then $I$ is a reflexive prime ideal. Let $\mathbb{U}$ be a parametric set of $I$ and $y_{i}$ all the other variables such that the characteristic set of $I$ under the variable order $\mathbb{U}<y_{i}$ is a coherent and strong irreducible chain $\mathcal{A}$. By [8], this is always possible. From Theorem 4.1, we may find $\sigma_{1}, \ldots, \sigma_{p} \in \mathcal{K}\{\mathbb{U}\}$ such that the characteristic set of $\operatorname{sat}\left(I, w-\sum_{i=1}^{p} \sigma_{i} y_{i}\right)$ under the variable order $\mathbb{U}<w<y_{i}$ is of the form (5). Let $W=\operatorname{Zero}\left(\operatorname{sat}\left(R, R_{1}, \ldots, R_{s}\right)\right)$. Then $W$ is an irreducible difference variety of codimension one. We define the following rational maps:

$$
\begin{aligned}
& M_{1}: V \Rightarrow W ;\left(\mathbb{U}, y_{1}, \ldots, y_{p}\right) \Rightarrow\left(\mathbb{U}, \sum_{i=1}^{p} \sigma_{i} y_{i}\right) \\
& M_{2}: W \Rightarrow V ;(\mathbb{U}, w) \Rightarrow\left(\mathbb{U}, \frac{V_{1}(\mathbb{U}, w)}{I_{1}(\mathbb{U}, w)}, \ldots, \frac{V_{p}(\mathbb{U}, w)}{I_{p}(\mathbb{U}, w)}\right)
\end{aligned}
$$

$M_{2}$ can be defined on $W-\operatorname{Zero}\left(\prod_{i} I_{i}\right)$. From the construction of $\sigma_{i}$, it is easy to show that $M_{1}$ and $M_{2}$ are inverse to each other. Hence $V$ and $W$ are birationally equivalent. I

We hence call sat $\left(R, R_{1}, \ldots, R_{s}\right)$ the resolvent ideal and $R, R_{1}, \ldots, R_{s}$ the resolvent system of $V$ or $I=\operatorname{sat}(\mathcal{A})$.

Note that from the results about resolvents in [2], we cannot obtain Corollary 4.2. The reason is that in [2], it is only proved that $I_{i} y_{i, m_{i}}-V_{i} \in I$ for some non-negative integer $m_{i}$. So the construction of $M_{2}$ is not valid. For the same reason, we also do not know whether the characteristic set of $\operatorname{sat}\left(\mathcal{A}, w-\sum_{i=1}^{p} \sigma_{i} y_{i}\right)$ is of the form (5). For instance, we do not know whether the characteristic set contains nonlinear r-pols in $y_{i, j}$.

\subsection{Algorithms to compute the resolvent system}

We first give two algorithms to compute the first element of the resolvent system for $\operatorname{sat}(\mathcal{A})$.

Algorithm 4.3 Input: a coherent and strong irreducible chain $\mathcal{A}$ of the form (1) and a variable set $\Lambda=\left\{\lambda_{1}, \ldots, \lambda_{p}\right\}$. Output: a $T \in \mathcal{K}\{\mathbb{U}, \Lambda, w\}$ which is an r-pol in $\operatorname{sat}(\mathcal{A}, w-$ $\left.\sum_{i=1}^{p} \lambda_{i} y_{i}\right)$ with the lowest rank w.r.t the variable order $\mathbb{U}<\lambda_{i}<w<y_{i}$.

Step 1 Let $o=\operatorname{ord}(\mathcal{A}), \mathcal{A}_{o}=\mathcal{A}_{(o, \ldots, o)}$, and $\mathbb{U}^{*}, \mathbb{Y}^{*}$ the sets of $u_{i j}$ and $y_{i j}$ occurring in $\mathcal{A}_{o}$ respectively. Then $\mathcal{A}_{o}$ is an irreducible algebraic triangular set in $\mathcal{K}\left[\mathbb{U}^{*}, \mathbb{Y}^{*}\right][8]$.

Step 2 Let $\mathcal{A}_{o}^{\prime}=\mathcal{A}_{o}, w_{0}-\sum_{i=1}^{p} \lambda_{i, 0} y_{i, 0}, \ldots, w_{o}-\sum_{i=1}^{p} \lambda_{i, o} y_{i, o}$ and $\Lambda_{o}=\left\{\lambda_{i, j}, i=1, \ldots, p ; j=\right.$ $0, \ldots, o\}$. Then it is clear that $\mathcal{A}_{o}^{\prime}$ is an irreducible algebraic triangular set in $\mathcal{K}\left[\Lambda_{o}, \mathbb{U}^{*}, \mathbb{Y}^{*}\right]$.

Step 3 Compute a characteristic set $\mathcal{C}$ of a-sat $\left(\mathcal{A}_{o}^{\prime}\right)$ in the polynomial $\operatorname{ring} \mathcal{K}\left[\Lambda_{o}, \mathbb{U}^{*}, \mathbb{Y}^{*}\right]$ under the variable order $\mathbb{U}<\lambda_{i, j}<w_{0}<w_{1}<\ldots<w_{o}<y_{i, j}$ with methods proposed in $[4,19]$ and output the first element in $\mathcal{C}$.

Proof of the correctness of Algorithm 4.3. Let $\mathcal{B}$ be a characteristic set of a-sat $\left(\mathcal{A}_{o}^{\prime}\right)$ under the given variable order. It is clear that $\mathbb{U}^{*}$ and $\Lambda_{o}$ are in the parametric set of a-sat $\left(\mathcal{A}_{o}^{\prime}\right)$. 
By the definition of the order for a chain, $\mathcal{A}_{o}^{\prime}$ has $o=\operatorname{ord}(\mathcal{A})$ parameters of the form $y_{i, j}$. In other words, a-sat $\left(\mathcal{A}_{o}^{\prime}\right)$ is of dimension $o$ over the base field $\mathcal{K}\left(\mathbb{U}^{*}, \Lambda_{o}\right)$. Since $w_{i}, i=0, \ldots, o$ are $o+1$ linear combinations of $y_{i, j}$, they must satisfy an algebraic relation. Let $T$ be such a relation and with the lowest rank. Then $T \in \operatorname{a-sat}\left(\mathcal{A}_{o}^{\prime}\right) \subset \operatorname{sat}\left(\mathcal{A}, w-\sum_{i=1}^{p} \lambda_{i} y_{i}\right)$. From Theorem 4.1, we know that the r-pol in $\operatorname{sat}\left(\mathcal{A}, w-\sum_{i=1}^{p} \lambda_{i} y_{i}\right)$ with the lowest rank under the variable order $\mathbb{U}<\Lambda<w<y_{i}$ is of order $o$ in $w$. Hence $T$ must involve $w_{o}$ and is an r-pol in $\operatorname{sat}\left(\mathcal{A}, w-\sum_{i=1}^{p} \lambda_{i} y_{i}\right)$ with the lowest rank w.r.t the variable order $\mathbb{U}<\lambda_{i}<w<y_{i}$.

The following algorithm uses the difference zero decomposition theorem to compute the first element of the resolvent system.

Algorithm 4.4 Input: a coherent and strong irreducible chain $\mathcal{A}$ of the form (1) and variables $\Lambda=\left\{\lambda_{i}, i=1, \ldots, p\right\}$. Output: $T \in \mathcal{K}\left\{\mathbb{U}, \lambda_{1}, \ldots, \lambda_{p}, w\right\}$ which is an r-pol in $\operatorname{sat}\left(\mathcal{A}, w-\sum_{i=1}^{p} \lambda_{i} y_{i}\right)$ with the lowest rank w.r.t the variable order $\mathbb{U}<\lambda_{i}<w<y_{i}$.

Step 1 Let $\mathcal{B}=\mathcal{A}, w-\sum_{i=1}^{p} \lambda_{i} y_{i}$. By Lemma 3.3, we have

$$
\operatorname{Zero}\left(\operatorname{sat}(\mathcal{B}) / \mathbf{I}_{\mathcal{A}}\right)=\operatorname{Zero}\left(\mathcal{B} / \mathbf{I}_{\mathcal{A}}\right)
$$

Step 2 Using the difference zero decomposition theorem proposed in [8], we may find the following zero decomposition under the variable order $\mathbb{U}<\lambda_{i}<w<y_{1}<\ldots<y_{p}$

$$
\operatorname{Zero}\left(\mathcal{B} / \mathbf{I}_{\mathcal{A}}\right)=\cup_{i} \operatorname{Zero}\left(\operatorname{sat}\left(\mathcal{A}_{i}\right) / \mathbf{I}_{\mathcal{A}}\right)
$$

where $\mathcal{A}_{i}$ are coherent and proper irreducible after a proper renaming of the variables. From (7) and the above equation, we have

$$
\operatorname{sat}(\mathcal{B})=\cap_{i}\left\{\operatorname{sat}\left(\mathcal{A}_{i}\right)\right\} .
$$

Step 3 By Theorem 4.1, a characteristic set of $\operatorname{sat}(\mathcal{B})$ if of the form (5). By Corollary 3.10, one of $\mathcal{A}_{i}$, say $\mathcal{A}_{1}$, must have $\mathbb{U}$ as its parametric set and $\operatorname{ord}\left(\mathcal{A}_{1}\right)=\operatorname{ord}(\mathcal{A})$. Let $T$ be the first element of $\mathcal{A}_{1}$. Then $T$ is in $\mathcal{K}\{\mathbb{U}, \Lambda, w\}$ and $\operatorname{ord}(T, w)=\operatorname{ord}(\mathcal{A})$. By Corollary 3.12, $T$ must also be the first element of the characteristic set of $\operatorname{sat}(\mathcal{B})$ under the variable order $U<\Lambda<w<y_{i}$. Output $T$.

Now, we give the following algorithm to compute the resolvent system.

Algorithm 4.5 Input: a coherent and strong irreducible chain $\mathcal{A}$ of the form (1). Output: $\sigma_{i} \in \mathcal{K}\{\mathbb{U}\}$ and a characteristic set of $\operatorname{sat}\left(\mathcal{A}, w-\sum_{i=1}^{p} \sigma_{i} y_{i}\right)$ which is of the form (5).

Step 1 Let $\lambda_{i}, i=1, \ldots, p$ be $p$ variables. With Algorithms 4.4 or 4.3, we may compute a $T \in \mathcal{K}\left\{\mathbb{U}, \lambda_{1}, \ldots, \lambda_{p}, w\right\}$ which is an $\mathrm{r}$-pol in $\operatorname{sat}\left(\mathcal{A}, w-\sum_{i=1}^{p} \lambda_{i} y_{i}\right)$ with the lowest rank w.r.t the variable order $\mathbb{U}<\lambda_{i}<w<y_{i}$.

Step 2 Let $H$ be the resultant of $\frac{\partial T}{\partial w_{0}}$ and $T$ w.r.t $w_{o}$. Find r-pols $A, B$ such that $A T+$ $B \frac{\partial T}{\partial w_{0}}=H$.

Step 3 For $i=1, \ldots, p$, let $Q_{i}=B\left(\frac{\partial T}{\partial \lambda_{i, 0}}+y_{i, 0} \frac{\partial T}{\partial w_{0}}\right)+A T y_{i, 0}=H y_{i, 0}+B \frac{\partial T}{\partial \lambda_{i 0}} \in \operatorname{sat}(\mathcal{A}, w-$ $\left.\sum_{i=1}^{p} \lambda_{i} y_{i}\right), H_{i}=\operatorname{rprem}\left(Q_{i}, T\right)$. 
Step 4 Let $\mathcal{B}=\mathcal{A}, w-\sum_{i=1}^{p} \sigma_{i} y_{i}$, and $Q \in \mathcal{K}\{\mathbb{U}, \lambda\}$ be the product of the coefficients of $\operatorname{rprem}\left(\operatorname{init}\left(H_{i}\right), \mathcal{B}\right)$ as a polynomial in $y_{i, j}$. By Lemma 2.1 , we may select $\sigma_{i} \in \mathcal{K}\{\mathbb{U}\}$ such that $Q\left(\sigma_{1}, \ldots, \sigma_{p}\right) \neq 0$.

Step 5 Let $P_{i}=\left.\left(H_{i}\right)\right|_{\left(\lambda_{1}, \ldots, \lambda_{p}\right)=\left(\sigma_{1}, \ldots, \sigma_{p}\right)}$. From the proof of Theorem 4.1, we know that $P_{i}=$ $I_{i} y_{i 0}-V_{i} \in \operatorname{sat}\left(\mathcal{A}, w-\sum_{i=1}^{p} \sigma_{i} y_{i}\right), I_{i} \notin \mathbf{s a t}\left(\mathcal{A}, w-\sum_{i=1}^{p} \sigma_{i} y_{i}\right)$, and $\operatorname{ord}\left(I_{i}, w\right)<\operatorname{ord}(\mathcal{A})$.

Step 6 Using the zero decomposition theorem proposed in [8], we may find the following decomposition under the variable order $\mathbb{U}<w<y_{1}<\ldots<y_{p}$

$$
\begin{aligned}
& \operatorname{Zero}\left(\operatorname{sat}\left(\mathcal{A}, w-\sum_{i=1}^{p} \sigma_{i} y_{i}, P_{1}, \ldots, P_{p}\right) / \mathbf{I}_{\mathcal{A}}\right) \\
= & \operatorname{Zero}\left(\mathcal{A} \cup\left\{w-\sum_{i=1}^{p} \sigma_{i} y_{i}, P_{1}, \ldots, P_{p}\right\} / \mathbf{I}_{\mathcal{A}}\right) \\
= & \cup_{i} \operatorname{Zero}\left(\operatorname{sat}\left(\mathcal{B}_{i}\right) / \mathbf{I}_{\mathcal{A}}\right)
\end{aligned}
$$

where $\mathcal{B}_{i}$ are coherent and proper irreducible after a proper renaming of the variables.

Step 7 Using the similar argument in Step 3 of Algorithm 4.4, there is a $\mathcal{B}_{i}$, which is of the form (5) and $\operatorname{ord}(R, w)=\operatorname{ord}(\mathcal{A})$. Then $\operatorname{sat}\left(\mathcal{A} \cup\left\{w-\sum_{i=1}^{p} \sigma_{i} y_{i}\right\}\right)$ must be the only prime component of $\left\{\boldsymbol{s a t}\left(\mathcal{B}_{i}\right)\right\}$. By Corollary $3.13, \mathcal{B}_{i}$ is strong irreducible and $\operatorname{sat}\left(\mathcal{A} \cup\left\{w-\sum_{i=1}^{p} \sigma_{i} y_{i}\right\}\right)=\operatorname{sat}\left(\mathcal{B}_{i}\right)$. Output $\mathcal{B}_{i}$.

In the above algorithm, we need to introduce $p$ new parameters, which will increase the computational costs. In the following, we will give a probability algorithm.

Theorem 4.6 Let $\mathcal{A}$ be a coherent and strong irreducible chain of the form (1) and $\sigma_{i}, i=$ $1, \ldots, p$ elements in $\mathcal{K}\{\mathbb{U}\}$. Using the zero decomposition theorem proposed in [8], we may find the following decomposition under the variable order $\mathbb{U}<w<y_{1}<\ldots<y_{p}$

$$
\operatorname{Zero}\left(\operatorname{sat}\left(\mathcal{A}, w-\sum_{i=1}^{p} \sigma_{i} y_{i}\right) / \mathbf{I}_{\mathcal{A}}\right)=\operatorname{Zero}\left(\mathcal{A} \cup\left\{w-\sum_{i=1}^{p} \sigma_{i} y_{i}\right\} / \mathbf{I}_{\mathcal{A}}\right)=\cup_{i} \operatorname{Zero}\left(\operatorname{sat}\left(\mathcal{B}_{i}\right) / \mathbf{I}_{\mathcal{A}}\right)
$$

where $\mathcal{B}_{i}$ are coherent and proper irreducible chains after a proper renaming of the variables. If one of $\mathcal{B}_{i}$, say $\mathcal{B}_{1}$, is of the form (5), then $\mathcal{B}_{1}$ is strong irreducible and is a resolvent system for $\operatorname{sat}(\mathcal{A})$.

Proof. Since $\mathcal{B}_{i}$ is coherent and proper irreducible, by Corollary 3.10, all its prime components $P_{i, j}, j=1, \ldots, m_{i}$, must have the same dimension and order with $\mathcal{B}_{i}$. From the zero decomposition, we have

$$
\operatorname{sat}\left(\mathcal{A}, w-\sum_{i=1}^{p} \sigma_{i} y_{i}\right)=\cap_{i}\left\{\operatorname{sat}\left(\mathcal{B}_{i}\right)\right\}=\cap_{i, j} P_{i, j}
$$

Since $P=\operatorname{sat}\left(\mathcal{A}, w-\sum_{i=1}^{p} \sigma_{i} y_{i}\right)$ is a prime ideal, there must exist $i_{0}, j_{0}$ such that $P_{i_{0}, j_{0}}=P$ and all other $P_{i, j} \subset P_{i_{0}, j_{0}}$. By corollary $3.13, \mathcal{B}_{i_{0}}$ is strong irreducible and $\operatorname{sat}\left(\mathcal{B}_{i_{0}}\right)=P_{i_{0}, j_{0}}$ is a prime ideal. Hence $\left\{\operatorname{sat}\left(\mathcal{B}_{i}\right)\right\} \subset \operatorname{sat}\left(\mathcal{B}_{i_{0}}\right)=P_{i_{0}, j_{0}}$ and $P=\operatorname{sat}\left(\mathcal{B}_{i_{0}}\right)$. From the above 
discussion, we can see that there exists only one $B_{i}$ which is with $\mathbb{U}$ as parameters and has order $\operatorname{ord}(\mathcal{A})$. Since $B_{1}$ is of the form $(5), \operatorname{sat}\left(B_{1}\right)$ is with $\mathbb{U}$ as parameters and has order $\operatorname{ord}(\mathcal{A})$. Then $B_{1}$ must be $B_{i_{0}}$ and hence a resolvent system for $\operatorname{sat}(\mathcal{A})$. I

Based on Theorem 4.6, we may just select $p$ elements $\sigma_{i}$ from $\mathcal{K}\{\mathbb{U}\}$ randomly and compute the zero decomposition (8). The probability of success is nearly one since by Theorem 4.1, the "bad" $\sigma_{i}$ are solutions of an r-pol equation $Q=0$.

Example 4.7 Consider the coherent and strong irreducible chain $\mathcal{A}=\left\{y_{1}^{2}+x, y_{2,1}^{2}+y_{2}^{2}+\right.$ $\left.1, y_{2,2}-y_{2}\right\}$. Let $w=y_{1}+y_{2}$. Using the zero decomposition theorem proposed in [8], we find the following decomposition under the variable order $w<y_{1}<y_{2}$ :

$$
\operatorname{Zero}\left(\operatorname{sat}\left(\mathcal{A}, w-y_{1}-y_{2}\right)\right)=\operatorname{Zero}\left(\mathcal{A} \cup\left\{w-y_{1}-y_{2}\right\}\right)=\operatorname{Zero}\left(\operatorname{sat}\left(\mathcal{B}_{1}\right)\right)
$$

where $\mathcal{B}_{1}$ is:

$$
\begin{aligned}
R= & w_{1}^{8}+\left(8+4 w^{2}\right) w_{1}^{6}+\left(16 w^{2}+6 w^{4}+8 x^{2}+16\right) w_{1}^{4}+\left(4 w^{6}+8 w^{4}+32 x^{2}-48 w^{2} x^{2}-64 w^{2} x\right) w_{1}^{2} \\
& +w^{8}+8 w^{4} x^{2}+16 x^{4}, \\
R_{1}= & \left(2 w_{1}^{2} w+2 w^{3}-4 w x\right) w_{2}^{2}+\left(-2 w_{1}^{2} w^{2}+w_{1}^{4}-3 w^{4}+4 w_{1}^{2}+4 x^{2}\right) w_{2} \\
& +4 w^{3}+w^{5}-8 w x-w w_{1}^{4}+4 x w^{3}-4 w x^{2}, \\
P_{1}= & \left(-4 w^{3}-4 w_{1}^{2} w+8 w x\right) y_{1}+4 w_{1}^{2}+2 w_{1}^{2} w^{2}+w^{4}+w_{1}^{4}-8 w^{2} x+4 x^{2}, \\
P_{2}= & \left(-8 w x+4 w_{1}^{2} w+4 w^{3}\right) y_{2}-3 w^{4}-2 w_{1}^{2} w^{2}+4 w_{1}^{2}+w_{1}^{4}+4 x^{2} .
\end{aligned}
$$

By Theorem 4.6, $\left\{R, R_{1}\right\}$ is the resolvent system for $\operatorname{sat}(\mathcal{A})$.

\section{Resolvent system of a proper irreducible chain}

Generally, we do not know how to decide wether a chain is strong irreducible or not. On the other hand, we can decide whether a chain is proper irreducible. In this section, we will give an algorithm to construct the resolvent system for the saturation ideal of a proper irreducible chain.

Theorem 5.1 Let $\mathcal{A}$ be a coherent and proper irreducible chain of the form (1) and $\lambda_{1}, \ldots, \lambda_{p}$ difference variables. We assume that $\mathcal{K}$ is an aperiodic difference field or $|\mathbb{U}| \neq 0$. Then there exists $Q \in \mathcal{K}\left\{\lambda_{1}, \ldots, \lambda_{p}, \mathbb{U}\right\}$ such that if $\sigma_{1}, \ldots, \sigma_{p}$ satisfy $Q\left(\sigma_{1}, \ldots, \sigma_{p}, \mathbb{U}\right) \neq 0$, the characteristic set of $\left\{\operatorname{sat}\left(\mathcal{A}, w-\sum_{i=1}^{p} \sigma_{i} y_{i}\right)\right\}$ under the variable order $\mathbb{U}<w<y_{i}$ is of the following form

$$
R, R_{1}, \ldots, R_{s}, I_{1} y_{1,0}-V_{1}, \ldots, I_{p} y_{p, 0}-V_{p}
$$

where $R, R_{i}, I_{i}, V_{i} \in K\{\mathbb{U}, w\}$. Furthermore, we have $R$ is effective in $w$ and $\operatorname{ord}(R, w)=$ $\operatorname{ord}(\mathcal{A})$.

Proof: Let $o=\operatorname{ord}(\mathcal{A}), Y_{h}=\left(y_{1,0}, y_{1,1}, \ldots, y_{1, h}, \ldots, y_{p, 0}, \ldots, y_{p, h}\right), W_{h}=\left(w_{0}, \ldots, w_{h}\right)$, and $\Lambda=\left\{\lambda_{1}, \ldots, \lambda_{p}\right\}$. For any r-pol set $\mathbb{P}$, we denote $\mathbb{P}^{\prime}=\left\{\mathbb{P}, w-\sum_{r}^{p} \lambda_{i=1} y_{i}\right\}$, that is, the perfect ideal generated by $\left\{\mathbb{P}, w-\sum_{i=1}^{p} \lambda_{i} y_{i}\right\}$. Let $\{\operatorname{sat}(\mathcal{A})\}=\bigcap_{s=1}^{r} P_{s}$ be an irredundant decomposition of $\{\operatorname{sat}(\mathcal{A})\}$ as the intersection of prime difference ideals. Then $\left\{\operatorname{sat}(\mathcal{A}), w-\sum_{i=1}^{p} \lambda_{i} y_{i}\right\}=\bigcap_{i=1}^{r} P_{i}^{\prime}$, where $P_{s}^{\prime}=\left\{P_{s}, w-\sum \lambda_{i} y_{i}\right\}$. By Theorem 4.1, for each 
$s$, the characteristic set for $P_{s}^{\prime}$ under the variable order $\mathbb{U}<\lambda_{i}<w<y_{i}$ is of the following form:

$$
T_{s}, T_{s, 1}, \ldots, T_{s, l_{s}}, I_{s, 1} y_{s, 0}-V_{s, 1}, \ldots, I_{s, p} y_{s, 0}-V_{s, p}
$$

where $T_{i}, T_{i, j}, I_{i, j}, V_{i, j} \in \mathcal{K}\{\mathbb{U}, w\}$ and $\operatorname{ord}\left(T_{s}, w\right)=o, \operatorname{ord}\left(I_{i, j}\right)<o$, and $\operatorname{ord}\left(V_{i, j}\right) \leq o$.

Let $P_{s}^{*}=P_{s}^{\prime} \bigcap \mathcal{K}\{\mathbb{U}, \Lambda\}\left[W_{o}, Y_{0}\right]$. Then $\mathbb{P}^{*}=\left\{\operatorname{sat}(\mathcal{A}), w-\sum_{i=1}^{p} \lambda_{i} y_{i}\right\} \cap \mathcal{K}\{\mathbb{U}, \lambda\}\left[W_{o}, Y_{0}\right]=$ $\bigcap_{s=1}^{r} P_{s}^{*}$. Also, $T_{s}, I_{s, j} y_{s, 0}-V_{s, j} \in P_{s}^{*}$. It is easy to see that $P_{s}^{*}=\mathbf{a}-\operatorname{sat}\left(T_{s}, I_{s, 1} y_{s, 0}-\right.$ $\left.V_{s, 1}, \ldots, I_{s, p} y_{s, 0}-V_{s, p}\right)$ is an algebraic prime ideal. From the proof of Theorem 4.1, we know that $I_{s, i} y_{s, i}-V_{s, i}$ are constructed from $T_{s}$. Thus if $T_{s}=T_{t}, P_{s}^{*}=P_{t}^{*}$. We rename the subscripts $s$, such that

$$
P_{1}^{*}=P_{2}^{*}=\ldots=P_{i_{1}-1}^{*}, \ldots, P_{i_{t}}^{*}=P_{i_{t}+1}^{*}=\ldots=P_{r}^{*}
$$

and $P_{1}^{*}, P_{i_{1}}^{*}, \ldots, P_{i_{t}}^{*}$ are not equal to each other. Denote $i_{0}=1$. We have $T_{i_{k}} \neq T_{i_{j}}$ for $k \neq j$. So $\mathbb{P}^{*}=\bigcap_{j=0}^{t} P_{i_{j}}^{*}$ is an irredundant representation and $S_{k}=\prod_{l=0}^{k} T_{i_{l}} \in \bigcap_{j=0}^{k} P_{i_{j}}^{*}$. We will show that there exist $I_{i}, V_{i} \in \mathcal{K}\{\mathbb{U}, \Lambda, w\}$, such that $I_{i} y_{i 0}-V_{i} \in \mathbb{P}^{*}$ and $\operatorname{ord}\left(I_{i}, w\right)<o$. We will construct such r-pols by doing induction on $k$. Suppose that there exists $J_{i, k} y_{i, 0}-W_{i, k} \in$ $\cap_{j=0}^{k} P_{i_{j}}^{*}$ and $\operatorname{ord}\left(J_{i, k}, w\right)<o$. Since $S_{k} \in \cap_{j=0}^{k} P_{i_{j}}^{*}$ and $Q_{k}=T_{i_{k+1}} \in P_{i_{k+1}}^{*}$ are difference rpols of the same order w.r.t $w$ and $Q_{k}$ is irreducible, the resultant $H$ of $S_{k}$ and $Q_{k}$ w.r.t $w_{o}$ must be nonzero and of order lower than $o$. From the property of the resultant, there exist $A(w), B(w) \in \mathcal{K}\{\mathbb{U}\}\left[W_{o}\right]$ such that $H=A S_{k}+B Q_{k}$. Let

$$
\begin{aligned}
J_{i, k+1} & =B Q_{k} J_{i, k}+A S_{k} I_{i, k+1}, \\
W_{i, k+1} & =B Q_{k} W_{i, k}+A S_{k} V_{i, k+1} .
\end{aligned}
$$

Then, we have

$$
\begin{aligned}
& J_{i, k+1} y_{i, 0}-W_{i, k+1}=A S_{k} I_{i, k+1} y_{i, 0}-A S_{k} V_{i, k+1}=H\left(I_{i, k+1} y_{i, 0}-V_{i, k+1}\right)=0 \bmod P_{i_{k+1}}^{*} \\
& J_{i, k+1} y_{i, 0}-W_{i, k+1}=B Q_{k} J_{i, k} y_{i, 0}-B Q_{k} W_{i, k}=H\left(J_{i, k} y_{i, 0}-W_{i, k}\right)=0 \bmod \cap_{j=0}^{k} P_{i_{j}}^{*}
\end{aligned}
$$

Therefore, $J_{i, k+1} y_{i, 0}-W_{i, k+1} \in \cap_{j=0}^{k+1} P_{i_{j}}^{*}$. Since $J_{i, k+1}=H I_{i, k+1} \neq 0 \bmod P_{i_{k+1}}^{*}$ and $J_{i, k+1}=$ $H J_{i, k} \neq 0 \bmod \cap_{j=0}^{k} P_{i_{j}}^{*}$, the resultant $H^{\prime}$ of $J_{i, k+1}$ and $S_{k+1}$ w.r.t $w_{o}$ is not zero. We have rpols $A^{\prime}, B^{\prime}$ such that $H^{\prime}=A^{\prime} J_{i, k+1}+B^{\prime} S_{k+1}$. Let $P_{i}=A^{\prime}\left(J_{i, k+1} y_{i, 0}-W_{i, k+1}\right)+B^{\prime} S_{k+1} y_{i, 0}=$ $H^{\prime} y_{i, 0}-A^{\prime} W_{i, k+1} \in \cap_{j=0}^{k+1} P_{i_{j}}^{*}$. Then there exists $P_{i}=I_{i} y_{i, 0}-V_{i} \in \mathbb{P}^{*} \subset\left\{\operatorname{sat}(\mathcal{A}), w-\sum \lambda_{i} y_{i}\right\}$. Let $Q=\prod_{i=1}^{p} I_{i}$. Since $\mathcal{K}\{\mathbb{U}\}$ is aperiodic, there exist $\sigma_{i} \in \mathcal{K}\{\mathbb{U}\}$, such that $\bar{Q}$, which is obtained by replacing $\lambda_{i}$ by $\sigma_{i}$ in $Q$, is not 0 . We have $\overline{I_{i}} \neq 0$ and $\bar{I}_{i} y_{i 0}+\bar{V}_{i} \in\{\operatorname{sat}(\mathcal{A}), w-$ $\left.\sum \sigma_{i} y_{i}\right\} \subset\left\{P_{i}, w-\sum \sigma_{i} y_{i}\right\}$. From the Theorem 4.1, the characteristic set of $\left\{P_{i}, w-\sum \sigma_{i} y_{i}\right\}$ under variable order $\mathbb{U}<w<y_{i}$ is of the following form

$$
S_{i}, S_{i, 1}, \ldots, S_{i, l_{i}}, I_{i, 1} y_{i, 0}-V_{i, 1}, \ldots, I_{s, p} y_{s, 0}-V_{s, p}
$$

where $S_{i}, S_{i, j}, I_{i, j}, V_{i, j} \in \mathcal{K}\{\mathbb{U}, w\}$ and $\operatorname{ord}\left(T_{s}, w\right)=o$. Let $R$ be the product of the different $S_{i}$. Then $R \in \cap_{i}\left\{P_{i}, w-\sum \sigma_{i} y_{i}\right\}=\left\{\operatorname{sat}(\mathcal{A}), w-\sum \sigma_{i} y_{i}\right\}$ and must be such an r-pol with lowest rank. Otherwise, let $H$ be an $r$-pol in $\left\{\operatorname{sat}(\mathcal{A}), w-\sum \sigma_{i} y_{i}\right\}$ with order $o$ in $w$ and 
lowest rank. Since $H \in\left\{P_{i}, w-\sum \sigma_{i} y_{i}\right\}$, we have $\operatorname{rprem}\left(H, S_{i}\right)=\operatorname{prem}\left(H, S_{i}\right)=0$. Since $S_{i}$ is irreducible, $S_{i}$ must be a factor of $H$. As a consequence $R$ is a factor of $H$, a contradiction. This proves the theorem. I

Unlike in Section 4., we cannot find the characteristic set for the resolvent system of $\operatorname{sat}(\mathcal{A})$ for a proper irreducible chain. On the other hand, we can find a resolvent system for $\operatorname{sat}(\mathcal{A})$.

Theorem 5.2 Let $\mathcal{A}$ be a coherent and proper irreducible chain of the form (1), $\mathcal{K}$ an aperiodic difference field or $|\mathbb{U}| \neq 0$. Then we can find $\sigma_{1}, \ldots, \sigma_{p} \in \mathcal{K}\{\mathbb{U}\}$ such that

$$
\begin{aligned}
\operatorname{Zero}\left(\operatorname{sat}\left(\mathcal{A}, w-\sum \sigma_{i} y_{i}\right)\right) & =\cup_{i=1}^{t} \operatorname{Zero}\left(\operatorname{sat}\left(\mathcal{R}_{i}\right)\right) \quad \text { or } \\
\left\{\operatorname{sat}\left(\mathcal{A}, w-\sum \sigma_{i} y_{i}\right)\right\} & =\cap_{i=1}^{t}\left\{\operatorname{sat}\left(\mathcal{R}_{i}\right)\right\}
\end{aligned}
$$

where each $\mathcal{R}_{i}$ is a coherent and proper irreducible chain under the variable order $\mathbb{U}<w<y_{i}$ and of the following form:

$$
R_{i}, R_{i, 1}, \ldots, R_{i, s_{i}}, I_{i, 1} y_{1,0}-V_{i, 1}, \ldots, I_{i, p} y_{p, 0}-V_{i, p},
$$

$R_{i}, R_{i, j}, I_{i, j}, V_{i, j} \in K\{\mathbb{U}, w\}$. Furthermore, $R_{i}$ is effective in $w$ and $\operatorname{ord}\left(R_{i}, w\right)=\operatorname{ord}(\mathcal{A})$.

We call $\left\{R_{i}, R_{i, 1}, \ldots, R_{i, s_{i}}\right\}, i=1, \ldots, t$, resolvent systems for $\operatorname{sat}(\mathcal{A})$. We will prove the theorem by giving an algorithm to compute the resolvent systems.

Algorithm 5.3 Input: a coherent and proper irreducible chain $\mathcal{A}$ of the form (1). Output: the resolvent systems for $\operatorname{sat}(\mathcal{A})$.

Step 1 Let $\mathcal{B}=\mathcal{A}, w-\sum_{i=1}^{p} \lambda_{i} y_{i}$. Then $\mathcal{B}$ is also a coherent and proper irreducible chain. By Lemma 3.3, we have $\operatorname{Zero}\left(\operatorname{sat}(\mathcal{B}) / \mathbf{I}_{\mathcal{A}}\right)=\operatorname{Zero}\left(\mathcal{B} / \mathbf{I}_{\mathcal{A}}\right)$.

Step 2 Using the difference zero decomposition theorem proposed in [8], under the variable order $\mathbb{U}<\lambda_{i}<w<y_{1}<\ldots<y_{p}$, we may find a decomposition

$$
\operatorname{Zero}\left(\operatorname{sat}(\mathcal{B}) / \mathbf{I}_{\mathcal{A}}\right)=\operatorname{Zero}\left(\mathcal{B} / \mathbf{I}_{\mathcal{A}}\right)=\cup_{i=1}^{s} \operatorname{Zero}\left(\operatorname{sat}\left(\mathcal{A}_{i}\right) / \mathbf{I}_{\mathcal{A}}\right)
$$

where $\mathcal{A}_{i}$ are coherent and proper irreducible after a proper renaming of the variables.

Step 3 Let $T_{i}$, and $P_{i, j}$ be the r-pols with lowest rank in $\mathcal{A}_{i}$ such that $\operatorname{lvar}\left(T_{i}\right)=w, \operatorname{lvar}\left(P_{i, j}\right)=$ $y_{j}$. By Theorem 4.1, the characteristic sets for all the prime components of $\operatorname{sat}(\mathcal{B})$ are of the form (5). By Corollary 3.10, $\left\{\boldsymbol{\operatorname { s a t }}\left(\mathcal{A}_{i}\right)\right\}$ is an unmixed ideal, and hence only for those $\mathcal{A}_{i}$ with $\mathbb{U}$ as the parametric set and satisfying $\operatorname{ord}\left(T_{i}, w\right)=\operatorname{ord}(\mathcal{A})$ and $\operatorname{ord}\left(P_{i, j}, y_{j}\right)=$ $0, \operatorname{Zero}\left(\operatorname{sat}\left(\mathcal{A}_{i}\right) / \mathbf{I}_{\mathcal{A}}\right)$ is not redundant. Remove all the $\operatorname{Zero}\left(\operatorname{sat}\left(\mathcal{A}_{i}\right) / \mathbf{I}_{\mathcal{A}}\right)$, where $\mathcal{A}_{i}$ does not satisfy the above condition. We may simply assume that all the $\mathcal{A}_{i}$ in (11) satisfy this condition.

Step 4 For each $\mathcal{A}_{i}$, let $S_{i, j}=\frac{\partial T_{i}}{\partial \lambda_{j}}+y_{j} \frac{\partial T_{i}}{\partial w_{0}}$. By Corollary 3.12, the first r-pol in the characteristic set for each prime component of $\left\{\operatorname{sat}\left(\mathcal{A}_{i}\right)\right\}$ is also $T_{i} . S_{i, j}$ is in each prime component of $\left\{\operatorname{sat}\left(\mathcal{A}_{i}\right)\right\}$ and hence $S_{i, j} \in\left\{\operatorname{sat}\left(\mathcal{A}_{i}\right)\right\}$. Let $R_{i}$ be the resultant of $\frac{\partial T_{i}}{\partial w_{0}}$ and $T_{i}$ w.r.t $w_{o}$. Then we may obtain r-pols $A$ and $B$ such that $R_{i}=A \frac{\partial T_{i}}{\partial w_{0}}+B T_{i}$. Let $Q_{i, j}=A S_{i, j}+B T_{i} y_{j, 0}=R_{i} y_{i, 0}+A \frac{\partial T_{i}}{\partial \lambda_{j 0}}$ and $P_{i, j}=\operatorname{rprem}\left(Q_{i, j}, T_{i}\right)$. We have $P_{i, j} \in\left\{\operatorname{sat}\left(\mathcal{A}_{i}\right)\right\}$. 
Step 5 Let $P_{i, j}=I_{i, j} y_{j, 0}-V_{i, j}$ where $I_{i, j}, V_{i, j} \in \mathcal{K}\{\mathbb{U}, \lambda, w\}$. Since $\operatorname{ord}\left(I_{i, j}, w\right)<\operatorname{ord}(\mathcal{A})$ and $\operatorname{ord}\left(T_{i}, w\right)=\operatorname{ord}(\mathcal{A}), I_{i, j}$ is not in each of $\operatorname{sat}\left(\mathcal{A}_{i}\right)$. Hence $I_{i, j}$ is invertible w.r.t $\mathcal{B}$. Let $Q_{0}$ be obtained by taking the successive resultant of $I_{i, j}$ and the r-pols in $\mathcal{B}_{I_{i, j}}$. Then $Q_{0}$ is not zero and in $\mathcal{K}\{\mathbb{U}\}[\mathcal{P}(\mathcal{A})]$, where $\mathcal{P}(\mathcal{A})$ is define $\operatorname{din}(3)$. Let $Q \in \mathcal{K}\{\mathbb{U}, \lambda\}$ be the product of the coefficients of $Q_{0}$ as a polynomial in $y_{i, j}$. Select $\sigma_{i} \in \mathcal{K}\{\mathbb{U}\}$ such that $Q\left(\sigma_{1}, \ldots, \sigma_{p}\right) \neq 0$.

Step 6 For an r-pol $P$ and a chain $\mathcal{C}$, let $\bar{P}$ and $\overline{\mathcal{C}}$ be obtained by replacing $\lambda_{i}$ with $\sigma_{i}$. It is clear that $\bar{P}_{i, j} \in\left\{\operatorname{sat}\left(\overline{\mathcal{A}}_{i}\right)\right\}$. Since $Q\left(\sigma_{1}, \ldots, \sigma_{p}\right) \neq 0, \bar{Q}_{0} \neq 0$. Then $\bar{I}_{i, j}$ is invertible w.r.t $\overline{\mathcal{B}}$ and hence not in $\operatorname{sat}(\overline{\mathcal{B}})$. Due to $(11), \bar{I}_{i, j} \notin\left\{\operatorname{sat}\left(\overline{\mathcal{A}}_{i}\right)\right\}$.

Step 7 Using the difference zero decomposition theorem proposed in [8], we may find the following decomposition under the variable order $\mathbb{U}<w<y_{1}<\ldots<y_{p}$

$$
\begin{aligned}
& \operatorname{Zero}\left(\operatorname{sat}\left(\overline{\mathcal{A}}_{i}\right) \cup\left\{\bar{P}_{i, 1}, \ldots, \bar{P}_{i, p}\right\} / J_{i}\right) \\
= & \operatorname{Zero}\left(\overline{\mathcal{A}}_{i} \cup\left\{\bar{P}_{i, 1}, \ldots, \bar{P}_{i, p}\right\} / J_{i}\right) \\
= & \cup_{j} \operatorname{Zero}\left(\operatorname{sat}\left(\mathcal{B}_{i, j}\right) / J_{i}\right)
\end{aligned}
$$

where $J_{i}$ is the product of initials of the r-pols in $\mathcal{A}_{i}$ and $\mathcal{B}_{i, j}$ are coherent and proper irreducible after a proper renaming of the variables.

Step 8 Using the similar argument in Step 3, we only select those $\mathcal{B}_{i, j}$ which are of the form (9). This is possible since $\bar{P}_{i, j}$ are linear in $y_{i, 0}$. Output $\mathcal{B}_{i, j}$. We have proved the Theorem 5.2.

Theorem 5.4 Let $\mathcal{A}$ be a coherent and proper irreducible chain of the form (1), $J$ the product of the initial of the r-pols in $\mathcal{A}$, and $\sigma_{i}, i=1, \ldots, p$ elements in $\mathcal{K}\{\mathbb{U}\}$. Suppose that we have the following zero decomposition under the variable order $\mathbb{U}<w<y_{1}<\ldots<y_{p}$

$$
\begin{aligned}
\operatorname{Zero}\left(\operatorname{sat}\left(\mathcal{A}, w-\sum \sigma_{i} y_{i}\right)\right) & =\cup_{i=1}^{t} \operatorname{Zero}\left(\operatorname{sat}\left(\mathcal{R}_{i}\right)\right) \quad \text { or } \\
\left\{\operatorname{sat}\left(\mathcal{A}, w-\sum \sigma_{i} y_{i}\right)\right\} & =\cap_{i=1}^{t}\left\{\operatorname{sat}\left(\mathcal{R}_{i}\right)\right\}
\end{aligned}
$$

where each $\mathcal{R}_{i}$ is a proper irreducible chain under the variable order $\mathbb{U}<w<y_{i}$ of the following form:

$$
R_{i}, R_{i, 1}, \ldots, R_{i, s_{i}}, I_{i, 1} y_{1,0}-V_{i, 1}, \ldots, I_{i, p} y_{p, 0}-V_{i, p}
$$

and $R_{i}, R_{i, j}, I_{i, j}, V_{i, j} \in K\{\mathbb{U}, w\}$. Furthermore, $R_{i}$ is effective in $w$ and $\operatorname{ord}\left(R_{i}, w\right)=\operatorname{ord}(\mathcal{A})$. Then $\left\{R_{i}, R_{i, 1}, \ldots, R_{i, s_{i}}\right\}$ are the resolvent systems for $\operatorname{sat}(\mathcal{A})$.

Proof. Similar to that of Theorem 4.6.

Example 5.5 Consider the chain $\mathcal{A}=\left\{y_{1}^{2}+x, y_{2,1}^{2}+y_{2}^{2}+1\right\}$. Let $w=y_{1}+y_{2}$. Using the zero decomposition theorem proposed in [8], we find the following decomposition under the variable order $w<y_{1}<y_{2}$ :

$$
\operatorname{Zero}\left(\operatorname{sat}\left(\mathcal{A}, w-y_{1}-y_{2}\right)\right)=\operatorname{Zero}\left(\mathcal{A} \cup\left\{w-y_{1}-y_{2}\right\}\right)=\operatorname{Zero}\left(\operatorname{sat}\left(\mathcal{B}_{1}\right)\right) \cup \operatorname{Zero}\left(\operatorname{sat}\left(\mathcal{B}_{2}\right)\right)
$$

where $\mathcal{B}_{2}=\left\{R, R_{1}^{\prime}, P_{1}, P_{2}\right\}, \mathcal{B}_{1}, R, P_{1}, P_{2}$ are given in Example 4.7 and $R_{1}^{\prime}=\left(-2 w_{1}^{2} w-\right.$ $\left.2 w^{3}+4 w x\right) w_{2}^{2}+\left(-2 w_{1}^{2} w^{2}+w_{1}^{4}-3 w^{4}+4 w_{1}^{2}+4 x^{2}\right) w_{2}$. It is clear that $\left\{R, R_{1}\right\}$ and $\left\{R, R_{1}^{\prime}\right\}$ are the resolvent systems for $\operatorname{sat}(\mathcal{A})$. 


\section{Conclusion}

Intuitively speaking, the theory of resolvent can be used to establish a birational correspondence between the solutions of a set of equations and the solutions of equations in one variable. For difference equations, the theory of resolvent is not complete in several aspects. In this paper, we give a more complete theory of difference resolvent systems. For an irreducible difference variety $V$, we can construct a coherent and strong irreducible chain $\mathcal{R}$ in one variable such that $V$ and $\operatorname{Zero}(\operatorname{sat}(\mathcal{R}))$ are birationally equivalent. For a coherent and proper irreducible chain $\mathcal{A}$, we can construct a series of coherent and proper irreducible chains $\mathcal{R}_{i}$ in one variable such that $\operatorname{Zero}(\operatorname{sat}(\mathcal{A}))$ and $\cup_{i} \operatorname{Zero}\left(\operatorname{sat}\left(\mathcal{A}_{i}\right)\right)$ are birationally equivalent.

An interesting problem is to see whether the union $\cup_{i} \operatorname{Zero}\left(\operatorname{sat}\left(\mathcal{A}_{i}\right)\right)$ in the proper irreducible case can be combined into one chain. That is, can we find a chain $\mathcal{B}$ such that $\cup_{i} \operatorname{Zero}\left(\operatorname{sat}\left(\mathcal{A}_{i}\right)\right)=\operatorname{Zero}(\operatorname{sat}(\mathcal{B}))$ ? To develop more efficient algorithms for difference resolvent systems is a very interesting and challenging problem.

\section{References}

[1] T. Cluzeau and E. Hubert, Resolvent Representation for Regular Differential Ideals, Applicable Algebra in Elementary Communication and Computing, 29, 395-425, 2003.

[2] R.M. Cohn, Difference Algebra, Interscience Pbulishers, 1965.

[3] R.M. Cohn, Manifolds of Difference Polynomials, Trans. of AMS, 64, 133-172, 1948.

[4] S.C. Chou, Mechanical Geometry Theorem Proving, D.Reidel Publishing Company, 1988.

[5] X.S. Gao and S.C. Chou, On the Parameterization of Algebraic Curves, Applicable Algebra in Elementary Communication and Computing, 3, 27-38, Springer-Verlag, 1992.

[6] X.S. Gao and S.C. Chou, On the Dimension for Arbitrary Ascending Chains, Chinese Bull. of Scis., vol. 38, 396-399, 1993.

[7] X.S. Gao and S.C. Chou, On the Theory of Resolvents and its Applications, Sys. Sci. and Math. Sci., 12, 17-30, 1999,

[8] X.S. Gao and Y. Luo, A Characteristic Set Method for Difference Polynomial Systems, International Conference on Polynomial System Solving, Nov. 24-26, 2004. Submitted to JSC.

[9] P. Gianni and T. Mora, Algebraic Solution of Systems of Polynomial Equations Using Gröbnert bases, Proc. of AAECC-5, pp. 247-257, LNCS, No. 356, Springer-Verlag, 1987.

[10] R. Loos, Computing in Algebraic Extensions, in Computer Algebra (Ed. by B. Buchberger, et al), 173-187, Springer-Verlag, New York, 1982.

[11] H. Kobayashi, S. Moritsugu and R.W. Hogan, Solving Systems of Algebraic Equations, Proc. of ISSAC-88, pp.139-149, LNCS No. 358, Springer-Verlag, 1988.

[12] E. Kolchin, Differential Algebra and Algebraic Groups, Academic Press, New York, 1973.

[13] E.L. Mansfield and A. Szanto, Elimination Theory for Differential Difference Polynomials, Proc. ISSAC 2002, 191-198, ACM Press.

[14] J.F. Ritt, Differential Algebra, Amer. Math. Soc. Colloquium, 1950.

[15] J.F. Ritt and J.L. Doob, Systems of Algebraic Difference Equations, American Journal of Mathematics, 55, 505-514, 1933 .

[16] B.M. Trager, Algebraic Factoring and Rational Integration, Proc. of ACM Sym. on Symbolic and Algebraic Computation, 1976. 
[17] D. Wang and D. Lin A Method for Multivariate Polynomial Factorization over Successive Algebraic Extension Fields, Mathematics and Mathematics Mechanization, 138-172, Shangdong Eductaional Press, 2001.

[18] J. van der Hoeven, Differential and Mixed Differential-difference Equations from the Effetive Viewpoint, Preprints, 1996.

[19] W.T. Wu, Basic Principle of Mechanical Theorem Proving in Geometries, (in Chinese) Science Press, Beijing, 1984; Springer, Wien, 1994.

[20] K. Yokoyama, M. Noro and T. Takeshima, Computing Primitive Elements of Extension Fields, J. Symbolic Computation, 8, 553-580, 1989. 